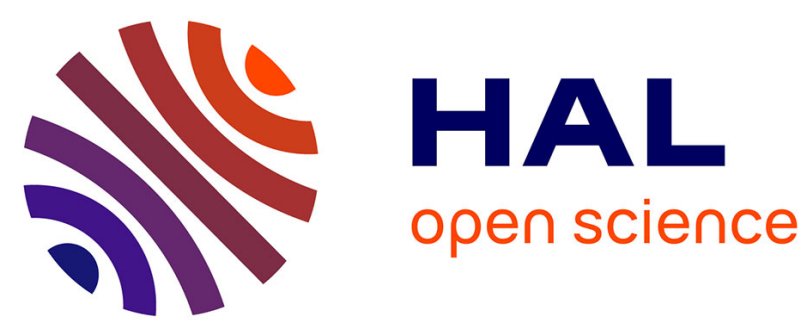

\title{
Ion hydration free energies and water surface potential in water nano drops: The cluster pair approximation and the proton hydration Gibbs free energy in solution
}

Céline Houriez, Florent Réal, Valérie Vallet, Michael Mautner, Michel Masella

\section{- To cite this version:}

Céline Houriez, Florent Réal, Valérie Vallet, Michael Mautner, Michel Masella. Ion hydration free energies and water surface potential in water nano drops: The cluster pair approximation and the proton hydration Gibbs free energy in solution. Journal of Chemical Physics, 2019, 151 (17), pp.174504. 10.1063/1.5109777 . hal-02355191

\author{
HAL Id: hal-02355191 \\ https://hal.science/hal-02355191
}

Submitted on 17 Nov 2020

HAL is a multi-disciplinary open access archive for the deposit and dissemination of scientific research documents, whether they are published or not. The documents may come from teaching and research institutions in France or abroad, or from public or private research centers.
L'archive ouverte pluridisciplinaire HAL, est destinée au dépôt et à la diffusion de documents scientifiques de niveau recherche, publiés ou non, émanant des établissements d'enseignement et de recherche français ou étrangers, des laboratoires publics ou privés. 


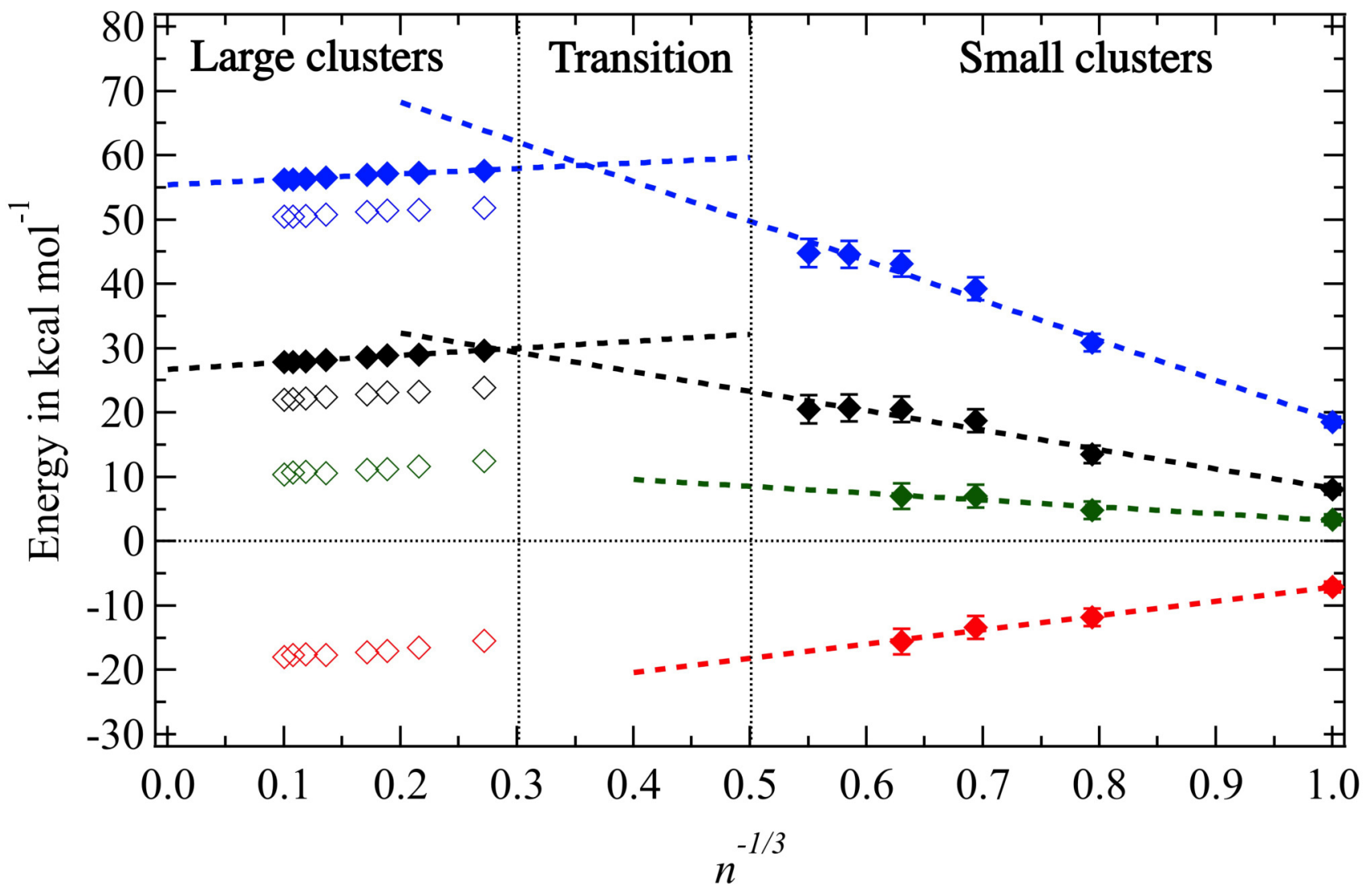




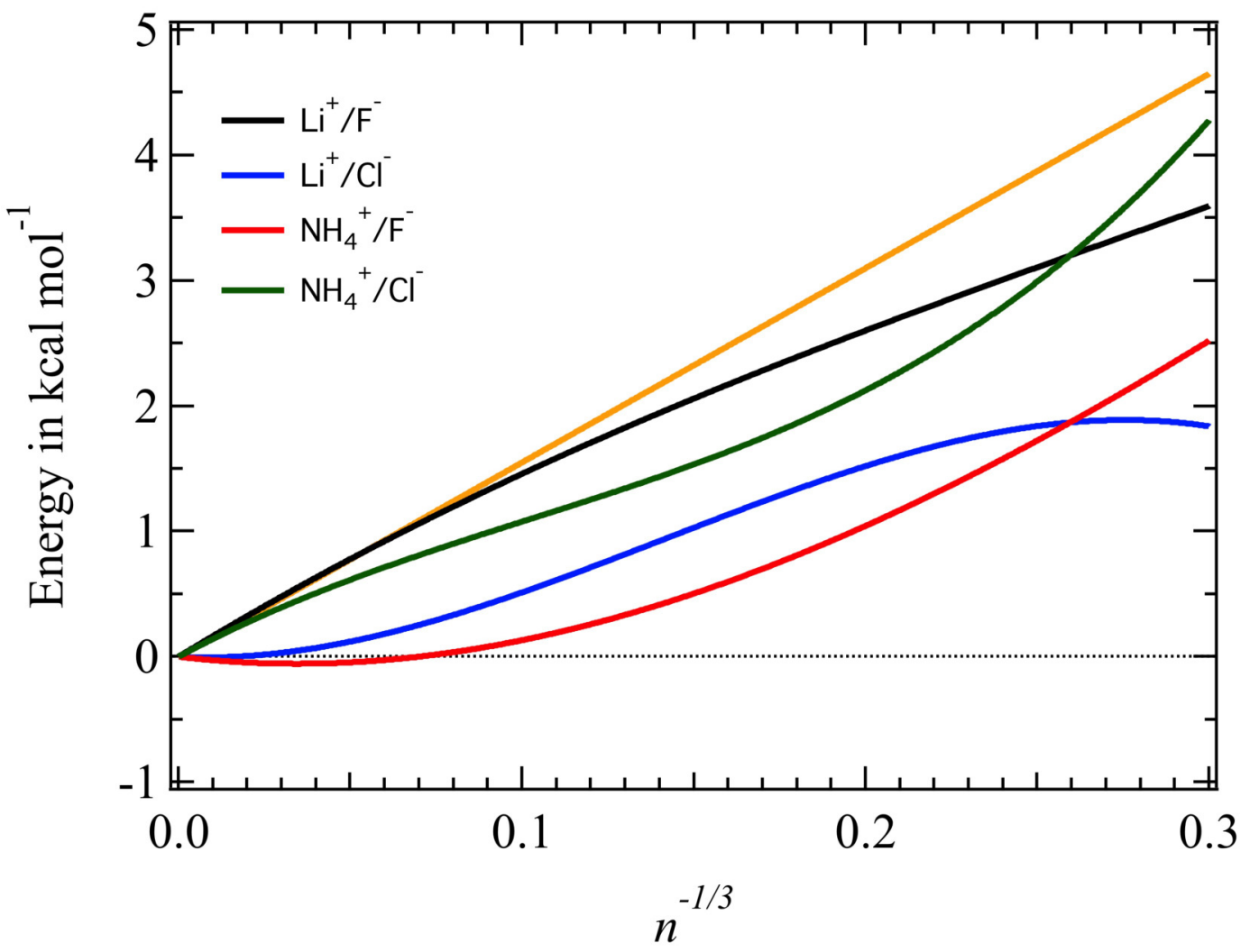




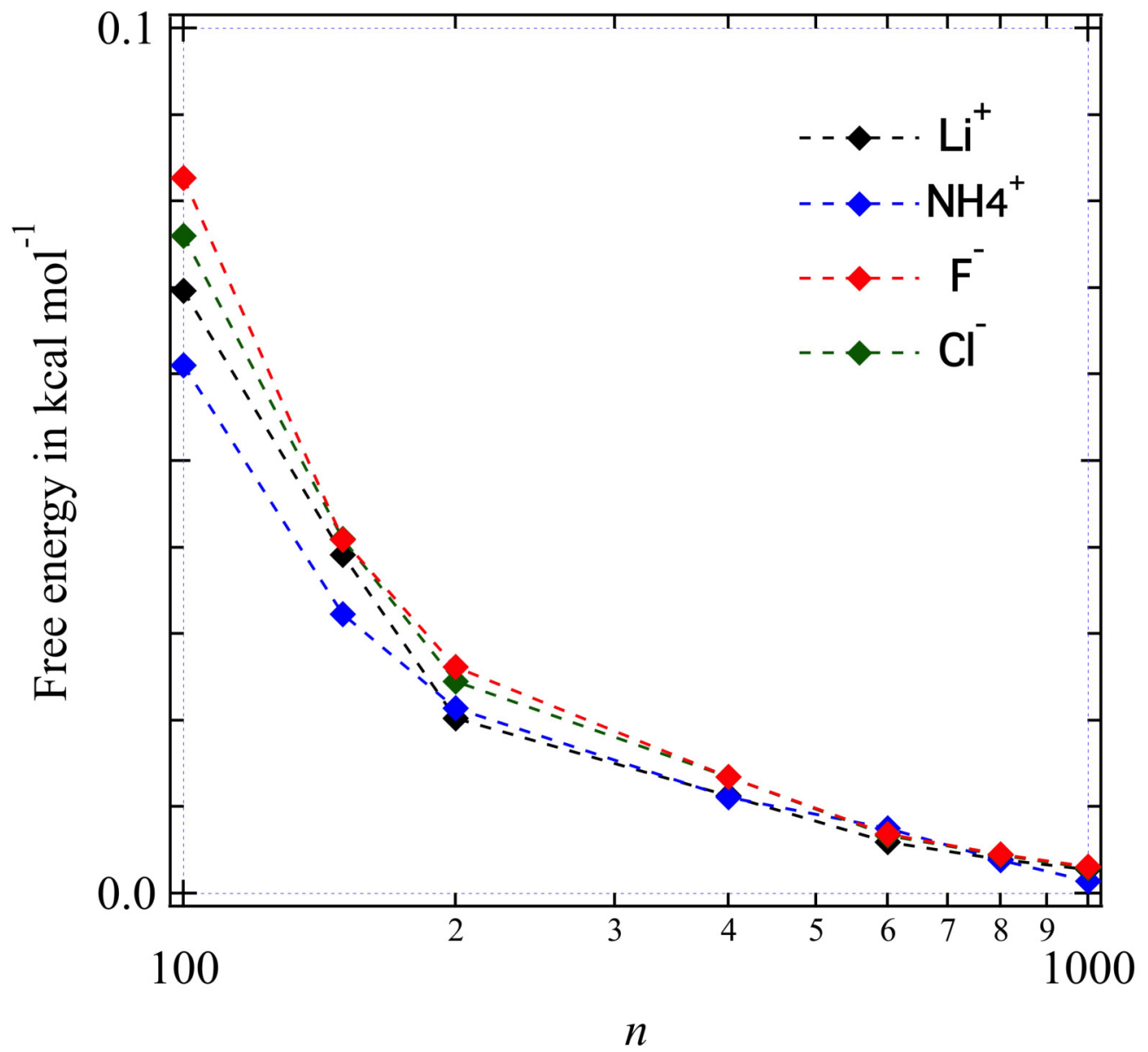




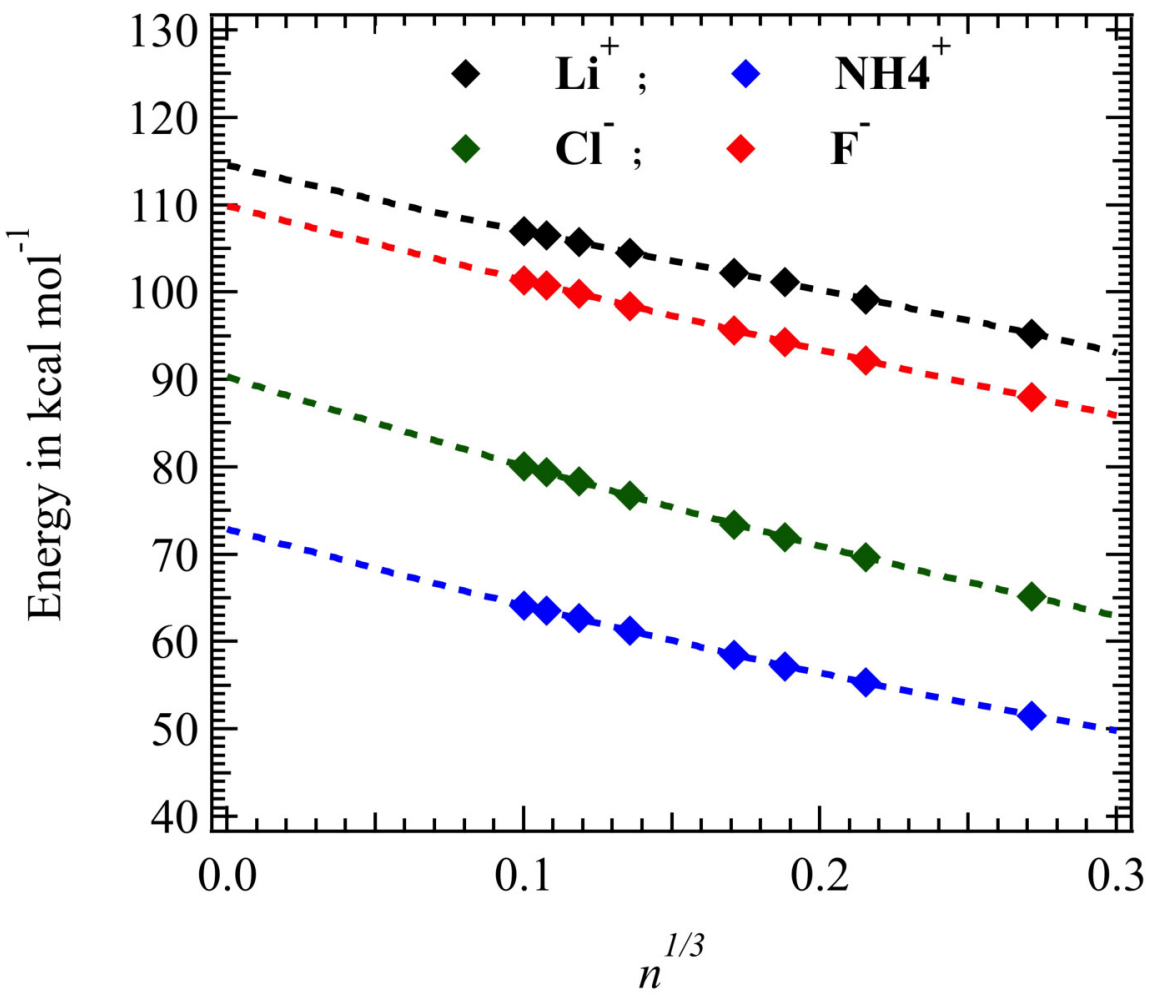




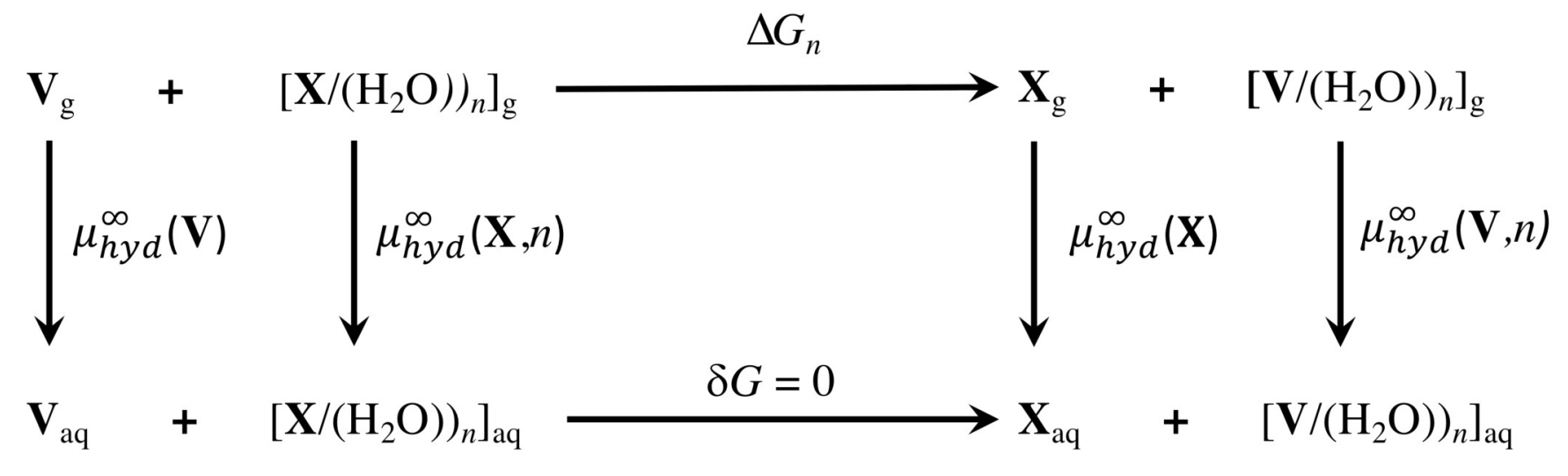




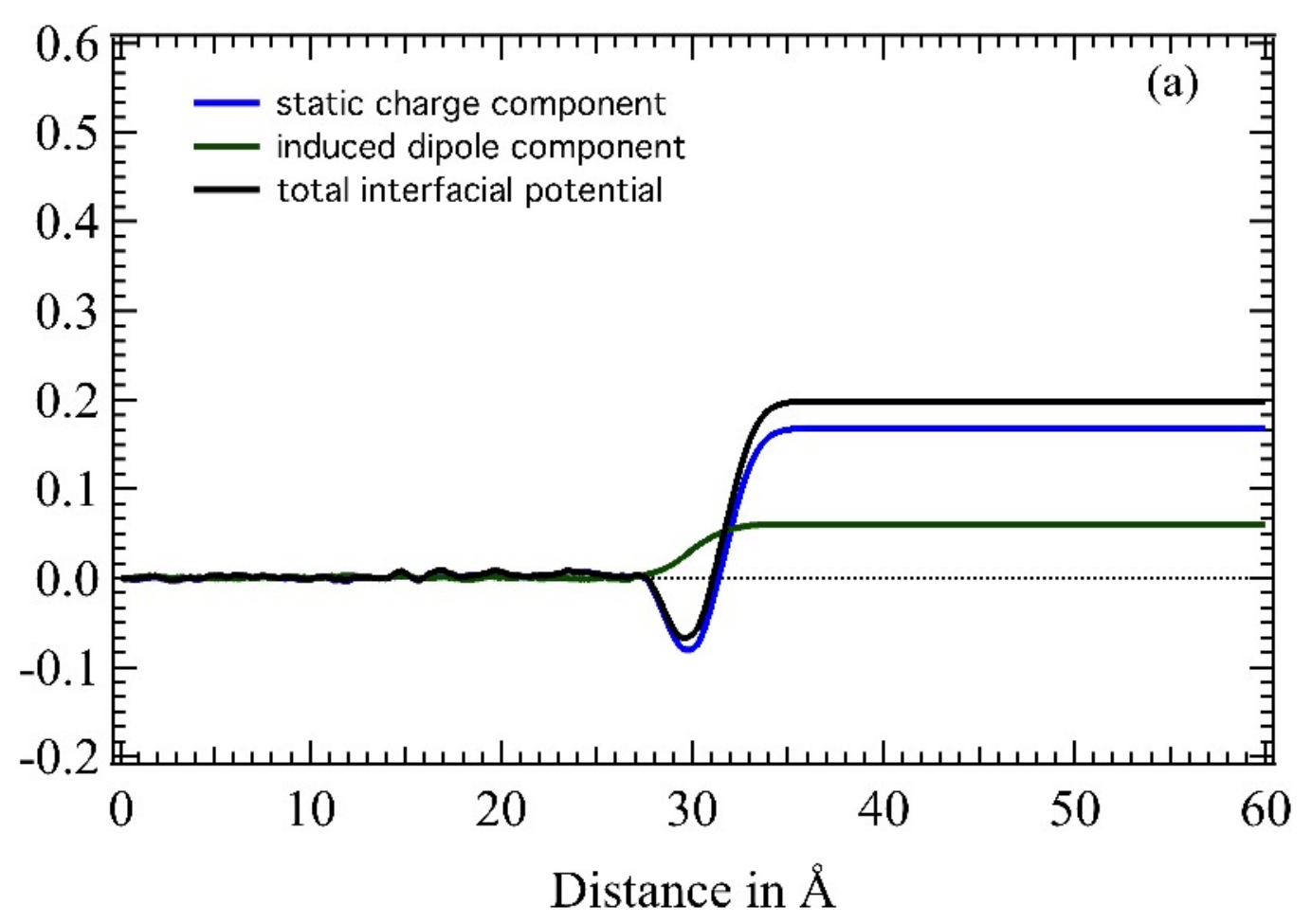




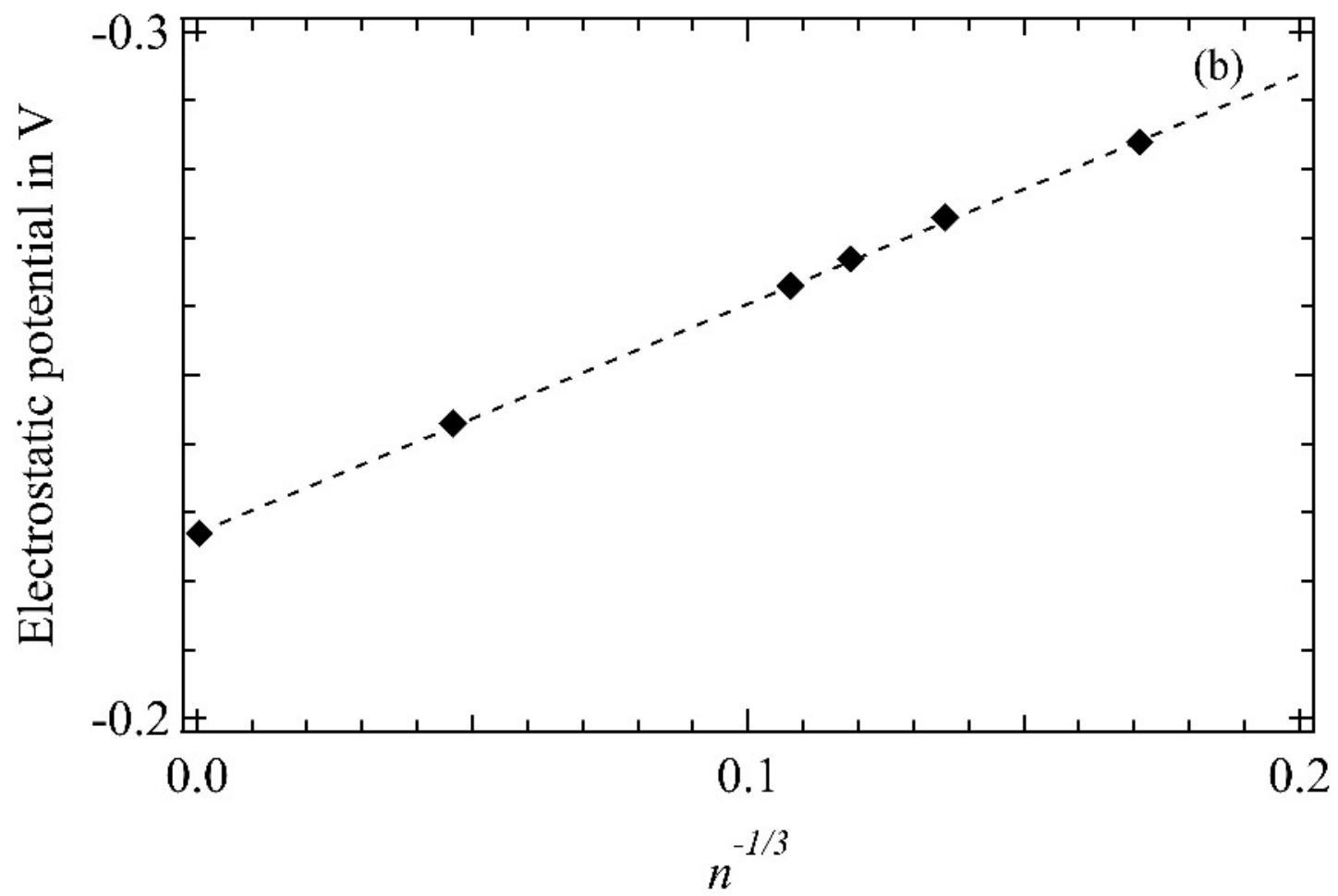




\section{Ion hydration free energies and water surface potential in water nano drops. The cluster pair approximation and the proton hydration Gibbs free energy in solution.}

Céline Houriez, ${ }^{\dagger}$ Florent Réal, ${ }^{\ddagger}$ Valérie Vallet, ${ }^{\ddagger}$ Michael Mautner, $₫$ and Michel Masella*,ई

$\dagger$ †INES ParisTech, PSL Research University, CTP - Centre Thermodynamique des Procédés, 35 rue Saint-Honoré, 77300 Fontainebleau, France

$\ddagger$ Univ. Lille, CNRS, UMR 8523 - PhLAM - Physique des Lasers Atomes et Molécules, F-59000 Lille, France

IDepartment of Chemistry, Virginia Commonwealth University, Richmond, Virginia 23284-2006, United States, and Department of Chemistry, University of Canterbury, Christchurch, New Zealand 8001

$\S$ Laboratoire de Biologie Structurale et Radiobiologie, Service de Bioénergétique, Biologie Structurale et Mécanismes, Institut Joliot, CEA Saclay, F-91191 Gif sur Yvette Cedex, France

E-mail: michel.masella@cea.fr 


\begin{abstract}
We estimate both single ion hydration Gibbs free energies in water droplets, comprising from 50 to 1000 molecules, and water/vacuum surface potentials in pure water droplets comprising up to 10000 molecules. We consider four ions, namely $\mathrm{Li}^{+}, \mathrm{NH}_{4}^{+}$, $\mathrm{F}^{-}$and $\mathrm{Cl}^{-}$and we model their hydration process and water/water interactions using polarizable force fields based on an induced point dipole approach. We show both ion hydration Gibbs free energies and water surface potentials to obey linear functions of the droplet radius as soon as droplets comprising a few hundred water molecules. Moreover we also show that the differences in anion/cation hydration Gibbs free energies in droplets obey a different regime in large droplets than in small clusters comprising no more than six water molecules, in line with earlier results computed from standard additive point charge force fields. Hence point charge and more sophisticated induced point dipole molecular modeling approaches both suggest that methods considering only the thermodynamical properties of small ion/water clusters to estimate the absolute proton hydration Gibbs free energy in solution are questionable. In particular taking into account the data of large ion/water droplets may yield a proton hydration Gibbs free energy in solution value to be shifted by several $k_{\mathrm{B}} \mathrm{T}$ units compared to small clusters-based approaches.
\end{abstract}




\section{9 \\ Introduction} $42 \mathrm{k}_{\mathrm{B}} \mathrm{T}$ at ambient conditions. ${ }^{9-13}$ Moreover only a few studies investigating the behavior of 43 water surface potential in water droplets have been reported, using standard additive water ${ }_{44}$ point charge models ${ }^{14,15}$ or a more sophisticated one, the AMOEBA approach, which includes 45

Ion hydration plays a pivotal role in many research fields from physics (like new high density batteries ${ }^{1}$ ), to chemistry (to understand pollution phenomena ${ }^{2}$ and climate change ${ }^{3}$ for instance), and biology. ${ }^{4,5}$ Despite decades of research, estimating ion hydration Gibbs free energies is far from being a routine activity. This arises from the difficulties with today experimental techniques to investigate the hydration process of a single ion in aqueous phase and from the necessity to consider theoretical models able to describe both short-range ion/water interactions, water structural perturbation arising from the ion presence and longrange ion/water interactions at an equal level of accuracy. This explains why until now the most accepted thermodynamical properties of the hydrated proton were computed from experimental and theoretical quantum data regarding only small ion/water clusters in gas phase (and whose size $n$ is no larger than 6 water molecules) and by assuming the Cluster Pair Assumption, CPA, i.e. the binding Gibbs free energies to add further water molecules to large enough anionic or cationic hydrated clusters become equal. ${ }^{6-8}$

Among long-range ion/water effects, we may quote the effect of water surface on ion hydration. The molecular organization at water boundaries can yield an unbalanced charge distribution and thus a difference in the electrostatic potential $\Delta \Phi$ between the water system core and its chemical environment (usually vacuum). $\Delta \Phi$ within a homogeneous phase is constant. It thus has no effect on ion dynamics within the bulk phase, however, it may favor/disfavor ion hydration by more or less largely shifting the ion hydration Gibbs free energy by a constant value $q \Delta \Phi$ ( $q$ being the ion charge). First of all we may note a large dispersion in the reported values of the water surface potential leading to a $q \Delta \Phi$ contribution to the hydration Gibbs free energy of an elementary charge $e$ ranging from -20 up to +140 dynamic polarization effects. ${ }^{16}$ Among these latter studies, we may quote in particular that 
68 free energy in solution. droplets. free energy in solution.

reported by Vlcek and co-authors who computed the absolute hydration enthalpies for a large set of monovalent and monoatomic ions dissolved in water droplets whose molecular sizes $n$ were $64,125,296$ and 1000 and by means of additive point charge force fields. ${ }^{15}$ Interestingly the latter authors concluded from their simulations that it is far from being obvious to consider small clusters-based methods to estimate the absolute proton hydration Gibbs free energy in solution as reliable and that significant differences may be expected from values computed when considering the thermodynamical properties of large sized ion/water

In the present study, we investigated the reliability of small clusters-based methods to estimate the proton hydration Gibbs free energy in solution by computing the absolute hydration Gibbs free energies of the monovalent ions $\mathrm{Li}^{+}, \mathrm{NH}_{4}^{+}, \mathrm{F}^{-}$and $\mathrm{Cl}^{-}$in aqueous phase as well as in isolated and finite size droplets whose molecular size as large as 1000 by means of molecular dynamics simulations based on polarizable force fields. We also estimated the surface potential in pure water droplets whose molecular size is as large as 10000 . The present manuscript is organized as follows. First we present the polarizable force fields, the numerical protocols used to compute the water surface potential and the absolute single ion hydration Gibbs free energies. Then we discuss the convergence of the water surface potential from droplets to bulk phase and the accuracy of our force fields to compute accurate absolute ion hydration Gibbs free energies from both finite and bulk phase ion/water systems. Lastly we discuss the relation between the difference in anion/cation hydration Gibbs free energies in droplets and the droplet surface potential and the consequence of that relation on the reliability of small clusters-based methods to estimate the absolute proton hydration Gibbs 


\section{${ }_{69} 1$ Methods and computational details}

70

71

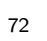

73

${ }_{84}$ All the $N_{\mu}$ non hydrogen atoms are polarizable centers, i.e. a single point polarizability is 85 located on each non-hydrogen atomic center. Their isotropic polarizability is $\alpha_{i}$. $\mathbf{E}_{i}^{q}$ is the 86

\subsection{The force field}

We use the rigid water model TCPE/ $2013^{17}$ and the ion/water force fields detailed in our former studies dealing about the hydration of $\mathrm{NH}_{4}^{+18}$ and of halide anions. ${ }^{19}$ Regarding the ion $\mathrm{Li}^{+}$we consider the ab-initio based force field detailed in Supplementary Material (see Section 1). The total potential energy $\Delta U$ corresponding to our force fields measures the total intermolecular interaction energy with respect to, wrt, individual unbound gas-phase chemical species. $\Delta U$ is a sum of five energy components

$$
\Delta U=U^{r e p}+U^{q q^{\prime}}+U^{d i s p}+U^{p o l}+U^{m b p} .
$$

For a system of $N$ atoms, the repulsive $U^{r e p}$, Coulombic $U^{q q^{\prime}}$ and dispersion $U^{d i s p}$ terms are defined as:

$$
U^{r e p}+U^{q q^{\prime}}+U^{d i s p}=\sum_{i=1}^{N} \sum_{j, j>i}^{N^{*}}\left[A_{i j} \exp \left(-B_{i j} r_{i j}\right)+\frac{q_{i} q_{j}}{4 \pi \varepsilon_{0} r_{i j}}-\left(\frac{\sigma_{i j}}{r_{i j}}\right)^{6}\right]
$$

here, $r_{i j}$ is the distance between atoms $i$ and $j,\left\{q_{i}\right\}$ are the static charges located on the atomic centers, and $\left(A_{i j}, B_{i j}, \sigma_{i j}\right)$ are adjustable parameters. Only cation/water interactions are modeled using dispersion. ${ }^{18}$ The superscript ${ }^{*}$ indicates the sum to include only atom pairs separated by more than two chemical bonds.

Polarization effects are modeled using induced dipole moments $\mathbf{p}_{i}$ that obey

$$
\mathbf{p}_{i}=\alpha_{i} \cdot\left(\mathbf{E}_{i}^{q}+\sum_{j=1}^{N_{\mu}^{*}} \mathbf{T}_{i j} \cdot \mathbf{p}_{j}\right)
$$

static electric field generated on the polarizable center $i$ by the surrounding charges $q_{j}$ and 
${ }_{87} \mathbf{T}_{i j}$ is dipolar tensor (they both include short-range Thole's-like damping functions ${ }^{17,20,21}$ ).

${ }_{88}$ The polarization energy is

$$
U^{p o l}=\frac{1}{2} \sum_{i=1}^{N_{\mu}} \frac{\mathbf{p}_{i}^{2}}{\alpha_{i}}-\sum_{i=1}^{N_{\mu}} \mathbf{p}_{i} \cdot \mathbf{E}_{i}^{q}-\frac{1}{2} \sum_{i=1}^{N_{\mu}} \sum_{j=1}^{N_{\mu}^{*}} \mathbf{p}_{i} \mathbf{T}_{i j} \mathbf{p}_{j} .
$$

89

$$
U^{3 b}=\sum A^{3 b} \exp \left(-B^{3 b} \times r_{\mathrm{O}_{\mathrm{i}} \mathrm{O}_{\mathrm{j}}}\right) f\left(r_{\mathrm{ClO}_{\mathrm{i}}}\right) f\left(r_{\mathrm{ClO}_{\mathrm{j}}}\right) .
$$

104 Here the sum runs on all the water oxygen pairs $(i, j)$ located at vicinity of anion and the 
function $f$ is defined as above for $U^{m b p} . A^{3 b}$ and $B^{3 b}$ are adjustable parameters that do not depend on the anion nature.

All the above force-field adjustable parameters allowing to model ion/water interactions are assigned to reproduce geometrical and energetic properties of small clusters as computed from high end quantum computations extrapolated to the complete basis set limit (see Refs $^{18,19,22}$ as well as Supplementary Material, Section 1). Note in particular our force fields for halides yield no overpolarization effect on the anion center as compared to the quantum CPMD approach. ${ }^{21}$ Regarding cations, we only consider small clusters where all the water oxygens interact directly with the cationic center.

\subsection{Molecular dynamics}

Our MD protocols correspond to those used in our former studies. ${ }^{18,23-25}$ Bulk phase systems (comprising all 1000 water molecules) are simulated in the NPT ensemble using periodic boundary conditions and the Smooth Particle Mesh Ewald, SPME, scheme devoted to polarizable force fields based on an induced dipole moment approach. ${ }^{26}$ To investigate the surface potential at the liquid water/vacuum interface, we simulate water slabs comprising 2000 molecules using periodic boundary conditions and the latter SPME scheme, but in the NVT ensemble. Droplet systems (comprising 50, 100, 150, 200, 400, 600, 800 and 1000 water molecules) are simulated in the NVT ensemble. To prevent evaporation phenomena, droplets are embedded in a large enough cavity and the interactions between the cavity wall and water molecules have no effect of the dynamics on the main droplet region that includes the water/vacuum interface. ${ }^{24}$ The water and $\mathrm{NH}_{4}^{+}$intramolecular degrees of freedom are constrained during the simulations. All simulations are performed at the $10 \mathrm{~ns}$ scale. Their last 9 ns segments are sampled each 1 ps to generate the statistical ensemble from which the simulation averages are computed. More details regarding our MD protocols are provided as Supplementary Material (see Section 4). 


\section{$1.3 \quad$ Surface potential}

The electrostatic potentials $\Phi$ at water-vacuum surfaces are computed by solving the Poisson's equation and by considering summations on atomic charge and dipole contributions (the so-called "P-convention" scheme)

$$
\nabla^{2} \Phi=-\frac{1}{\epsilon_{0}} \sum_{i=1}^{N}\left(q_{i}+\mathbf{p}_{i} \cdot \nabla_{i}\right) \delta\left(\mathbf{r}-\mathbf{r}_{i}\right)
$$

here, $\left(\mathbf{r}_{i}, q_{i}, \mathbf{p}_{i}\right)$ are the atomic position, static charge and induced dipole moment of atom $i$. $\delta$ is the volume delta function. As all the sized- $n$ droplets discussed here are quasi-spherical (see our former studies ${ }^{18}$ and below) we integrate the above equation in the droplet case as

$$
\Phi_{d}^{n}(r)=-\frac{1}{\epsilon_{0}} \int_{0}^{r} \frac{C_{q}^{n}(r)+C_{p}^{n}(r)}{r^{2}} d r
$$

The distance $r$ is measured from the droplet center of mass $(\mathrm{COM}) . C_{q}^{n}(r)$ is the temporal mean sum of the static charges included in a sphere of radius $r$ and $C_{p}^{n}(r)$ is the temporal mean density at distance $r$ of the projection of the induced dipoles in the direction orthogonal to the surface. In the liquid water-vacuum interface case, the above integral is rewritten as

$$
\Phi_{\text {bulk }}(z)=-\frac{1}{S \epsilon_{0}} \int_{0}^{z}\left(C_{q}(z)+C_{p}(z)\right) d z
$$

here $z$ is the distance from the simulated slab center in the direction orthogonal to the liquid water-vacuum interface. The functions $C_{q}$ and $C_{p}$ are computed by considering a rectangular domain orthogonal to the interface (whose surface $S$ is constant along NVT simulations).

Both the above integrals are computed discretely by setting $d r$ and $d z$ to $0.1 \AA$.

To compute accurate droplet surface potentials $\Phi_{d}^{n}(r)$, we performed 50 independent MD simulations (each of $10 \mathrm{~ns}$ and corresponding to a different set of initial atomic velocities) of pure water droplets comprising 200, 400, 600 and 800 molecules. The liquid water-vacuum surface potential $\Phi_{\text {bulk }}(z)$ is computed from 10 independent MD simulations (each also of 
$10 \mathrm{~ns}$ ) of a periodic slab system comprising 2000 water molecules. The functions $\bar{C}_{q / p}$ are the mean $C_{q / p}$ ones averaged on the independent MD trajectories and the $\delta C_{q / p} \mathrm{~s}$ are their corresponding root mean square deviation among the latter trajectories. We assume the $\delta C_{q / p}$ s to be a measure of the $\bar{C}_{q / p}$ uncertainties. They are smaller than $1 \%$, regardless of the water system considered. We also estimated the surface potential of a large water droplet made of 10000 molecules, however from a single trajectory generated as detailed in Ref. ${ }^{24}$

Note that we reported an erroneous $\Delta \Phi_{\text {bulk }}$ value for the water model TCPE/2013 in our earlier study. ${ }^{19}$ The error arises from an erroneous factor 2 introduced in the computations of the function $C_{q}(z)$. We check our corrected computational protocol by reproducing the $\Delta \Phi_{\text {bulk }}$ value for the water model TIP3P (about $0.5 \mathrm{~V},{ }^{27}$ see Supplementary Material, Figure 14).

We also computed the TCPE/2013 dipolar contribution $\Delta \Phi_{b u l k}^{\mathrm{D}}$ to the water surface potential by means of the so-called "M-convention" scheme, i.e. by considering the total molecular dipole vector as hosted at the water oxygen to solve the Poisson's equation. We checked the sum of the dipolar contribution $\Delta \Phi_{b u l k}^{\mathrm{D}}$ and of the Bethe's potential contribution $\Delta \Phi_{b u l k}^{\mathrm{Q}}$ arising from the water molecular quadrupole tensor to well reproduce the total water surface potential $\Delta \Phi_{\text {bulk }}$ (see Supplementary Material, Figure 14).

\subsection{Droplet and bulk Gibbs free energies}

To compute the single ion hydration Gibbs free energy, we consider the thermodynamic cycle TC shown in Figure 1. Based on the ion charge sign, we consider two different virtual, neutral and non polarizable atoms $\mathbf{V}$ whose first hydration shell structure in water is close that of a cation (i.e. $\mathbf{V}$ interacts directly with water oxygens) or of an anion ( $\mathbf{V}$ interacts directly with water hydrogens). We denote these two kinds of virtual atoms as $\mathbf{V}_{\mathrm{c}}$ and $\mathbf{V}_{\mathrm{a}}$ when needed. The water $/ \mathbf{V}$ interaction potentials were built to favor their presence within the droplet core along droplet NVT trajectories. The corresponding force-field parameters are provided in Supplementary Material (see Section 7, Table 3) and the propensity of both 
virtual atoms for the core of any size water droplets is shown by the centripetal character of their potential of mean force, PMF, as interacting with water droplets computed using a standard Umbrella Sampling scheme (see Supplementary Material, Section 7 and Figures 6 and 7).

According to the thermodynamic cycle $\mathbf{T C}$, the hydration Gibbs free energy of an ion $\mathbf{X}$ at infinite dilution is

$$
\mu_{\text {hyd }}^{\infty}(\mathbf{X})=\mu_{\text {hyd }}^{\infty}(\mathbf{V})-\Delta G_{n}+\left(\mu_{\text {hyd }}(\mathbf{X}, n)-\mu_{\text {hyd }}(\mathbf{V}, n)\right)
$$

Here $\Delta G_{n}$ is the Gibbs free energy cost corresponding to the alchemical reaction $\mathbf{X}_{\text {hyd }} \rightarrow \mathbf{V}_{\text {hyd }}$ in a sized- $n$ water droplet, $\mu_{\text {hyd }}^{\infty}(\mathbf{A})$ and $\mu_{\text {hyd }}(\mathbf{A}, n)$ are the absolute bulk hydration Gibbs free energies of the entity $\mathbf{A}$ in bulk water and of $\mathbf{A}$ embedded in sized- $n$ water droplet, respectively.

The quantity $\mu_{\text {hyd }}(\mathbf{A}, n)$ is the sum of three components: the Gibbs free energy cost $\mu_{\text {hyd }}^{\text {cav }}(\mathbf{A}, n)$ to create a cavity within liquid water and corresponding to the sized- $n$ cluster, the electrostatic cost $\mu_{\text {hyd }}^{\text {elec }}(\mathbf{A}, n)$ to embed the cluster in the latter cavity and the repulsion/hydrogen bond contribution $\mu_{\text {hyd }}^{\text {surfe }}(\mathbf{A}, n)$ corresponding to water/water local interactions at the cluster/cavity interface. We show that ion and $\mathbf{V}$ droplet systems are all quasi spherical and the difference in their volume between ion and $\mathbf{V}$ systems is negligible for the droplet systems that we consider (see Supplementary Material, Figure 5). The difference in the $\mu_{\text {hyd }}^{\text {cav }}(\mathbf{A}, n)$ and $\mu_{\text {hyd }}^{\text {surface }}(\mathbf{A}, n)$ contributions between ion and $\mathbf{V}$ droplets is thus assumed to vanish as $n \rightarrow \infty$ and Equation 11 may be rewritten for large enough droplets as

$$
\mu_{\text {hyd }}^{\infty}(\mathbf{X})=\mu_{\text {hyd }}^{\infty}(\mathbf{V})-\Delta G_{n}+\left(\mu_{\text {hyd }}^{\text {elec }}(\mathbf{X}, n)-\mu_{\text {hyd }}^{\text {elec }}(\mathbf{V}, n)\right)
$$


cavity (of radius $a_{n}$ ) within a continuum medium, the component $\mu_{\text {hyd }}^{\text {elec }}(\mathbf{A}, n)$ should obey ${ }^{28}$

$$
\mu_{\mathrm{hyd}}^{\mathrm{elec}}(\mathbf{A}, n)=\frac{1}{8 \pi \epsilon_{0}} \sum_{l=0}^{\infty} \frac{(l+1)(1-\epsilon)}{(l+1) \epsilon+l}\left(\frac{M_{l}}{a_{n}^{2 l+1}}\right),
$$

here $M_{l}$ is the electric moment of order $l$ of the charge distribution. That relation is still valid when considering a distribution of induced dipole moments as they can be modeled by a specific set of charges. However the above relation implicitly corresponds to an abrupt transition in the dielectric constant between the solute cavity and the solvent. For an ion solvated in aqueous phase, the water local dielectric permittivity $\epsilon(\mathbf{r})$ behavior is shown to be strongly oscillatory until a distance of $12 \AA$ from the ion. ${ }^{29}$ From the work of Beveridge and Schnuelle, ${ }^{28}$ that yields the $\mu_{\text {hyd }}^{\text {elec }}(\mathbf{A}, n)$ expansion for an ion to also include an $a_{n}^{-2}$ term.

We showed the molecular density within pure water droplets to be converged to the bulk value within less than $2 \%{ }^{18}$ The radius $a_{n}$ of a sized- $n$ droplet may be thus assumed to be proportional to $n^{-1 / 3}$ and from all the above, the gas-phase cluster $\Delta G_{n}$ may be written as a power law function of the droplet size $n$

$$
\Delta G_{n}=\Delta G_{\infty}+\frac{\gamma_{1}}{n^{1 / 3}}+\frac{\gamma_{2}}{n^{2 / 3}}+O\left(\frac{1}{n}\right)
$$

and according to Equation 11 the quantity $\Delta G_{\infty}$ is

$$
\Delta G_{\infty}=\lim _{n \rightarrow \infty} \Delta G_{n}=\mu_{\text {hyd }}^{\infty}(\mathbf{V})-\mu_{\text {hyd }}^{\infty}(\mathbf{X})
$$

\subsection{Computing Gibbs free energies}

The bulk hydration Gibbs free energies $\mu_{\text {hyd }}^{\infty}(\mathbf{A})$ are estimated from MD simulations in bulk phase using periodic boundary conditions and the Thermodynamic Integration (TI) scheme by progressively decoupling solute/solvent interactions. For ionic species, we compute their $\mu_{\text {hyd }}^{\infty}(\mathbf{X})$ in two steps, first by decoupling the electrostatic and polarization ion $\mathbf{X} /$ water interactions and then by decoupling the remaining interactions between the uncharged and 
non-polarizable entity $\mathbf{X}^{0}$ and water (i.e. interactions corresponding to the energy terms $U^{r e p}, U^{d i s p}$ and $\left.U^{s l h}\right)$.

We systematically consider a 20 windows TI scheme. Each window corresponds to a 10 ns MD simulation where the solute $\mathbf{A} /$ water solvent hamiltonian is scaled by a constant $\lambda$ regularly spaced between 1 and 0 . Each 50 MD steps, we compute the derivative of the system total potential energy $\Delta U$ wrt the scaling parameter $\lambda$ using a finite difference method. From the mean derivative values and their corresponding root mean square deviations, we compute both the integrals providing $\mu_{\text {hyd }}^{\infty}(\mathbf{A})$ and their uncertainties assuming that the derivatives computed along each MD windows are temporally uncorrelated. Because of the large size of the derivative data sets, the latter uncertainties are negligible, at most about $\pm 0.05 \mathrm{kcal}$ $\mathrm{mol}^{-1}$. Regarding the virtual atoms $\mathbf{V}$, their quantities $\mu_{\text {hyd }}^{\infty}(\mathbf{V})$ computed as above are -12.5 $\left(\mathbf{V}_{\mathrm{c}}\right)$ and $-9.0\left(\mathbf{V}_{\mathrm{a}}\right) \pm 0.05 \mathrm{kcal}^{\mathrm{mol}}{ }^{-1}$. Note here that these values agree with those that may be estimated form the virtual atom/water droplet PMFs provided as Supplementary Material (see Figures 6 and 7).

To remove periodic artifacts arising from accounting for ion images in our simulation

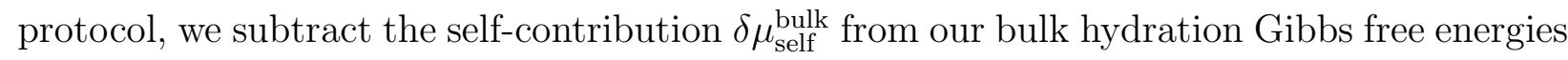
$\mu_{\text {hyd }}^{\infty}(\mathbf{X})$. As the solute static charges are scaled by the parameter $\lambda$ in our implementation of the TI scheme, $\delta \mu_{\text {self }}^{\text {bulk }}$ obeys

$$
\delta \mu_{\mathrm{self}}^{\text {bulk }}=\frac{\xi q^{2}}{8 \pi \epsilon_{0} \epsilon \bar{L}}
$$

$\epsilon$ is the liquid water dielectric constant predicted by the water model TCPE/2013 at 300 $\mathrm{K}\left(78.3^{17}\right)$ and $\bar{L}$ is the average simulation cell dimension along the NPT bulk trajectories, about $31 \AA$, and $\xi=2.837297$. That yields $\delta \mu_{\text {self }}^{\text {bulk }}=0.2 \mathrm{kcal} \mathrm{mol}^{-1}$. A priori we should also account for other periodic effects arising from the inappropriate orientational polarization of the solvent during the simulation, for instance. These corrections, corresponding to higher order terms of $\bar{L}^{-1}$, are here fully negligible because of the size of our simulation boxes. ${ }^{30}$

Regarding clusters, we compute their quantity $\Delta G_{n}$ from NVT droplet simulations and by means of the same TI scheme detailed above for bulk systems. We tested two approaches: 
they correspond (1) to alchemically transform the charged ion $\mathbf{X}$ in its corresponding virtual atom $\mathbf{V}$ in one step, and (2) to uncouple first the electrostatic and polarization ion/water interactions and then to alchemically transform the uncharged and non-polarizable entity $\mathbf{X}^{0}$ in its corresponding virtual atom $\mathbf{V}$. Both approaches provide equal results regarding the hydration Gibbs free energy in droplet, within less than $0.1 \mathrm{kcal} \mathrm{mol}^{-1}$.

\subsection{Further corrections to simulation-based estimate of ion hydra- tion Gibbs free energy}

To estimate reliable single ion bulk hydration Gibbs free energy from our simulation data, two physical contributions have also to be considered. The first, $\delta G_{\text {ref }}$, arises from the identical ion concentration in gas phase and in liquid water in our bulk phase simulation protocol, while experimentally the ion concentration in gas phase correspond to that of 1 mole of an ideal gas and to $1 \mathrm{M}$ in liquid water. We thus estimate $\delta G_{\text {ref }}$ as the amount of energy to compress 1 mole of an ideal gas to reach a concentration of $1 \mathrm{~mol} \mathrm{l^{-1 }}$. At ambient conditions, $\delta G_{\mathrm{ref}}=\mathrm{RT} \ln 22.4=+1.89 \mathrm{kcal} \mathrm{mol}^{-1}$.

The second, $\delta G_{v i b}^{g \rightarrow l}$, arises from the use of constrains in our MD simulations, i.e. intramolecular degrees of freedom of the non-monoatomic species $\mathrm{NH}_{4}^{+}$and $\mathrm{H}_{2} \mathrm{O}$ are frozen along our MD trajectories. However, quantum computations show large shifts in the intramolecular harmonic vibrational frequencies of $\mathrm{NH}_{4}^{+}$between its gas-phase isolated state and hydrated clusters. For the four hydrated $\mathrm{NH}_{4}^{+}$cluster, these shifts are on average of $-230 \mathrm{~cm}^{-1}$ for the cation harmonic stretching frequencies and of $+60 \mathrm{~cm}^{-1}$ for the bending ones. $^{31}$

Liquid water intramolecular vibrational frequencies may be also altered when water molecules interact at short range from an ion. For instance the mean stretching frequency $\nu_{\mathrm{OD}}$ in liquid HDO is experimentally reported to be shifted by about -70 and $+60 \pm 10 \mathrm{~cm}^{-1}$ compared to bulk as HDO molecules lie in the first hydration shell of $\mathrm{Li}^{+}$and $\mathrm{F}^{-}, 32$ respectively, in agreement for $\mathrm{Li}^{+}$with a quantum-based theoretical estimate. ${ }^{33}$ For both the anion 
$\mathrm{Cl}^{-}$and the cation $\mathrm{K}^{+}$, the latter experimental shift is small about $+20 \pm 10 \mathrm{~cm}^{-1}$ (cf. the data discussed in Ref. ${ }^{32}$ ). Regarding $\mathrm{Cl}^{-}$, we may quote also an ab initio molecular dynamics study ${ }^{34}$ showing the infrared stretching vibration spectrum of water molecules lying at its vicinity to be more dissymmetric than in the bulk (with a slightly larger weight of weak $\nu_{\mathrm{OH}}$ frequencies). In all that suggests a very weak effect of the $\mathrm{Cl}^{-} / \mathrm{K}^{+}$presence on the liquid water intramolecular vibrational properties. As the hydration properties of $\mathrm{NH}_{4}^{+}$are close to $\mathrm{K}^{+}$, we assume as negligible the enthalpic $\delta H_{v i b}^{g \rightarrow l}$ and Gibbs free energy $\delta G_{v i b}^{g \rightarrow l}$ costs arising from the perturbation of the liquid water intramolecular vibrational spectrum due to the $\mathrm{Cl}^{-} / \mathrm{NH}_{4}^{+}$presence.

We estimate the magnitude of $\delta G_{v i b}^{g \rightarrow l}$ (and of its enthalpic $\delta H_{v i b}^{g \rightarrow l}$ counterparts) arising from the solute or solvent intramolecular frequency shifts from standard polyatomic ideal gas formula (see Supplementary Material, Section 5). The enthalpy cost $\delta H_{v i b}^{g \rightarrow l}$ is estimated to be -0.8 and $-1.6 \mathrm{kcal} \mathrm{mol}^{-1}$ for $\mathrm{Li}^{+}$and $\mathrm{NH}_{4}^{+}$, respectively. The magnitude of the Gibbs free energy cost $\delta G_{v i b}^{g \rightarrow l}$ is slightly weaker, about -0.5 and $-1.0 \mathrm{kcal} \mathrm{mol}^{-1}$ for the latter two ions, respectively. The order of magnitude of these contributions supports the reliability of simulating ion hydration by constraining intramolecular degrees of freedom.

Hence adding the latter two corrections to $\mu_{\text {hyd }}^{\infty}(\mathbf{X})$ yields the absolute single ion bulk hydration Gibbs free energy value $\tilde{\mu}_{\text {hyd }}^{\infty}(\mathbf{X})$

$$
\tilde{\mu}_{\text {hyd }}^{\infty}(\mathbf{X})=\mu_{\text {hyd }}^{\infty}(\mathbf{X})+\delta G_{v i b}^{g \rightarrow l}+\delta G_{\text {ref }}
$$

\section{Results and discussion}

\subsection{Convergence of the water droplet surface potential to the bulk} limit

The plots of the $n$-sized droplet surface potential profiles $\Phi_{d}^{n}(r)$ are provided as Supplementary Material (see Figures 10 to 13). Agreeing with earlier results, ${ }^{11}$ their main features are 
similar to the bulk-vacuum profile $\Phi_{\text {bulk }}(z)$ shown in Figure $2(\mathrm{a}): \Phi_{d}^{n}(r)$ are constant outside of the droplets and within their cores, with a rapid transition at the droplet surfaces. We denote hereafter $\Delta \Phi_{d}^{n} / \Delta \Phi_{\text {bulk }}$ the difference in the $\Phi_{d}^{n}(r) / \Phi_{\text {bulk }}(z)$ values between vacuum and the aqueous core. As shown in Figure 2(b), $\Delta \Phi_{d}^{n}$ obeys a decreasing linear function of $n^{-1 / 3}$

$$
\Delta \Phi_{d}^{n}=\Delta \Phi_{\text {bulk }}-\frac{\phi}{n^{1 / 3}} .
$$

It converges towards the bulk limit $\Delta \Phi_{d}^{\infty}=-0.227 \mathrm{~V}$ that matches our computed $\Delta \Phi_{\text {bulk }}$ value within less than $0.1 \%$ (the slope $-\phi$ is here $-0.331 \mathrm{~V}$ ). Hence all the droplet values $\Delta \Phi_{d}^{n}$ are larger in magnitude than the bulk one, by $20 \%$ for the 200 sized droplet, for instance.

The linear dependence on $n^{-1 / 3}$ of our droplet $\Delta \Phi_{d}^{n}$ data agrees with earlier results reported by Kastenholz and Hünnenberger ${ }^{14}$ and by Vlcek and co-authors ${ }^{15}$ from simulations performed using the point charge water models SPC and SPC/E, respectively. However we may quote that Pollard and Beck ${ }^{16}$ showed the $\Delta \Phi_{d}^{n}$ values estimated from ion hydration enthalpy data in small droplets $(n<200)$ and the AMOEBA force field to exhibit also a decreasing but non $n^{-1 / 3}$ linear behavior.

The bulk surface potential value $\Delta \Phi_{\text {bulk }}$ predicted by the water model TCPE/2013 is less than half the value reported for most of the available water models ${ }^{11}$ based on point charges (point dipoles) approaches, but twice as large as the point-charge water model TIP5P. ${ }^{35}$ However the TCPE/2013 dipolar $\Delta \Phi_{\text {bulk }}^{\mathrm{D}}$ contribution is clearly larger than that corresponding to available water models including TIP5P and closer to the quantum DFT estimates, ${ }^{35}+0.38$ and $+0.48 \mathrm{~V}$, respectively. From the discussions reported by Remsing et al (in particular regarding their data summarized in the Table 2 in Ref. ${ }^{35}$ ) the latter result suggests TCPE/2013 to predict a molecular organization at the water surface close to that predicted by quantum DFT-based simulations. On the other hand and as all point charge-based models, TCPE/2013 yields an overall large but negative Bethe's potential contribution $\Delta \Phi_{\text {bulk }}^{\mathrm{Q}}=-0.61 \mathrm{~V}$, whereas quantum DFT simulations predict a very large and positive contribution, about $+4 \mathrm{~V} \cdot{ }^{35,36}$ 


\subsection{Mean stepwise water binding free energy in large ion/water droplets}

We define the mean stepwise water binding free energy $\delta \tilde{\mu}_{m \rightarrow n}$ as the free energy cost to further add a water molecule to ion $\mathbf{X}$ /water droplets whose size is included between $m$ and $n:$

$$
\delta \tilde{\mu}_{m \rightarrow n}=\frac{\mu_{\mathrm{hyd}}(\mathbf{X}, n)-\mu_{\mathrm{hyd}}(\mathbf{X}, m)}{n-m} .
$$

As shown by the plots of Figure 3, that quantity is about equal for our four ions no sooner than $m=400$. The specific nature of a monovalent ion has thus still a noticeable effect on ion/water interactions in aqueous droplets until a distance from the ion of about $R_{\delta \mu}=14 \AA$, a distance that matches the $R_{\epsilon}$ one for which the oscillatory behavior of the water permittivity induced by ion presence vanishes. ${ }^{29}$ As the two distances $R_{\delta \mu}$ and $R_{\epsilon}$ have been estimated using two different kinds of force field (in particular, a classical and a polarizable one), their agreement shows the influence of a specific ion on water to extend far beyond the usual cut off distance used by protocols based on the Quasi Chemical Theory (about $6 \AA^{37}$ ) to define the "inner-shell" term that is shown to produce alone accurate estimates of the full bulk ion hydration Gibbs free energy. However we may quote an experimental study that shows monovalent ions (namely $\mathrm{I}^{-}$and $\mathrm{Na}^{+}$) dissolved in $n \approx 250$ water droplets to not affect the water hydrogen bond network behind the ion second hydration shell. ${ }^{38}$

\subsection{Single ion hydration Gibbs free energies : from droplets to} bulk

As our force-field total potential energy $\Delta U$ is a sum of five components, we may decompose $\Delta G_{n}$ into five contributions

$$
\Delta G_{n}=\Delta G_{n}^{\mathrm{rep}}+\Delta G_{n}^{\mathrm{disp}}+\Delta G_{n}^{\mathrm{mbp}}+\Delta G_{n}^{\mathrm{qq}{ }^{\prime}}+\Delta G_{n}^{\mathrm{pol}} .
$$


The first three contributions correspond to interaction energy terms that are short ranged. As shown by our TI computations, they converge more rapidly to their bulk limit value than the last two contributions. The free energy components corresponding to the alchemical reaction $\left[\mathrm{NH}_{4}^{0}\left(\mathrm{Li}^{0}\right) /\left(\mathrm{H}_{2} \mathrm{O}\right)_{\mathrm{n}}\right] \rightarrow\left[\mathbf{V}_{\mathrm{c}},\left(\mathrm{H}_{2} \mathrm{O}\right)_{\mathrm{n}}\right]$ or to the sum of components $\Delta G_{n}^{\mathrm{rep}}+\Delta G_{n}^{\mathrm{disp}}+\Delta G_{n}^{\mathrm{mbp}}$ for the reaction $\left[\mathrm{Cl}^{-},\left(\mathrm{H}_{2} \mathrm{O}\right)_{\mathrm{n}}\right] \rightarrow\left[\mathrm{V}_{\mathrm{a}},\left(\mathrm{H}_{2} \mathrm{O}\right)_{\mathrm{n}}\right]$ are already converged within $0.25 \mathrm{kcal} \mathrm{mol}^{-1}$ on average as soon as $n=400$ and within $0.1 \mathrm{kcal} \mathrm{mol}^{-1}$ at $n=1000$, regardless of the ion. We denote both the latter quantities as $\Delta G_{n}^{\mathrm{sr}}$ and their converged $\Delta G_{\infty}^{\mathrm{sr}}$ values are summarized in Table 1.

We thus extrapolated only the sum of the non-zero electrostatic and polarization contributions $\Delta G_{n}^{\mathrm{elec}}=\Delta G_{n}^{\mathrm{qq}^{\prime}}+\Delta G_{n}^{\mathrm{pol}}$ using the power law function shown in Equation (14) for our four ions. The $\Delta G_{n}^{\text {elec }}$ quantities are plotted as functions of $n^{-1 / 3}$ in Figure 4 and the extrapolated $\Delta G_{\infty}^{\text {elec }}$ values are summarized in Table 1 . The uncertainty affecting the $\Delta G_{\infty}^{\text {elec }}$ values arising from the fitting process is $\leq 0.3 \mathrm{kcal} \mathrm{mol}^{-1}$. Accounting also for the uncertainties regarding the energy derivative averages computed along the TI MD simulations and the $\Delta G_{\infty}^{\mathrm{sr}}$ values yields a total error for the values $\Delta G_{\infty}=\Delta G_{\infty}^{\mathrm{sr}}+\Delta G_{\infty}^{\text {elec }}$ of about $\epsilon_{\mathrm{err}} \approx 0.6$ kcal $\mathrm{mol}^{-1}$. This supports the reliability our droplet-based protocol to compute single ion hydration thermodynamic properties.

In Table 1 we summarize the bulk limit values $\Delta G_{\infty}$ extrapolated from droplet data, the single ion bulk hydration Gibbs free energy $\mu_{\text {hyd }}^{\infty}(\mathbf{X})$ values computed from bulk simulations, the differences $\mu_{\text {hyd }}^{\infty}(\mathbf{X})-\mu_{\text {hyd }}^{\infty}(\mathbf{V})$ in the bulk hydration Gibbs free energy between ions and their corresponding virtual atoms. As expected from Equation (12), the difference in the quantities $\Delta G_{\infty}$ and $\left[\mu_{\text {hyd }}^{\infty}(\mathbf{V})-\mu_{\text {hyd }}^{\infty}(\mathbf{X})\right]\left(5.2 \pm 0.5 \mathrm{kcal} \mathrm{mol}^{-1}\right.$ on average and in absolute values) for all the ions equals the surface potential contribution $q \Delta \Phi_{\text {bulk }}$ within our computational protocol uncertainty.

We also estimated single ion bulk hydration Gibbs free energies from droplet data using different extrapolation schemes than above. For instance, we extrapolated the total Gibbs 
free energy $\Delta G_{n}=\Delta G_{n}^{\text {elec }}+\Delta G_{n}^{\text {sr }}$ using the power law function

$$
\Delta G_{n}=\Delta G_{\infty}^{\circ}+\frac{\gamma_{1}^{\circ}}{n^{1 / 3}}+\frac{\gamma_{2}^{\circ}}{n^{2 / 3}}+\frac{\gamma_{3}^{\circ}}{n} .
$$

The last term of the right hand equation is introduced to account for short range non electrostatic ion/water interactions. It is a priori well suited to account for ion/water dispersion as modeled in our force-field for cations $\mathrm{Li}^{+}$and $\mathrm{NH}_{4}^{+}$. The adjusted parameters $\Delta G_{\infty}^{\circ}$ and $\gamma_{1,2,3}^{\circ}$ are provided as Supplementary Material (see Table 4). The new single ion bulk hydration Gibbs free energy estimates $\Delta G_{n}^{\infty, 0}$ differ from those computed based on the Table 1 data by about $\pm 1 \mathrm{kcal} \mathrm{mol}^{-1}$, a difference in line with the uncertainty $\epsilon_{\text {err }}$ of computed Gibbs free energy data from our TI simulations.

As discussed in Section 2.3, the nature of a monovalent ion has an effect on water in aqueous droplets up to a distance of about $14 \AA$ from the ion center. Such a distance corresponds to the radius of a $n=400$-sized water droplet, a droplet size for which all the non electrostatic contributions to the single ion hydration Gibbs free energy in droplets are converged to their bulk limit, like $\Delta G_{n}^{\text {sr }}$ and $\mu_{\text {hyd }}(\mathbf{V}, n)$. Hence for $n \geq 400$, we may approximate a water droplet to a continuous medium. Assuming the dielectric constant of water droplets corresponding to $n \geq 400$ as already large enough ( $>10$, see Ref. $\left.{ }^{39}\right)$ and from the Born equation, the electrostatic free energy contribution $\mu_{\text {hyd }}^{\text {elec }}(\mathbf{X}, n)$ and thus the free energy $\Delta G_{n}$ should then obey a linear $n^{-1 / 3}$ regime

$$
\Delta G_{n}=\Delta \tilde{G}_{\infty}+\frac{\tilde{\gamma}}{n^{1 / 3}}
$$

The corresponding adjusted parameters are listed in Table 2. Note first the linear regression coefficients to be all $>0.99$ and even $>0.999$ for $\mathrm{Li}^{+}, \mathrm{F}^{-}$and $\mathrm{Cl}^{-}$supporting the assumption regarding the linear $n^{-1 / 3}$ regime to which the $\Delta G_{n}$ values (and thus the single ion hydration Gibbs free energies in droplets $\mu_{h y d}^{n}$ ) should obey in large droplets. Moreover and as their $\Delta G_{n}^{\infty, \circ}$ counterparts, the $\Delta \tilde{G}_{\infty}$ values agree with those that may be computed from the data 
listed in Table 1 also within $\pm 1 \mathrm{kcal} \mathrm{mol}^{-1}$ on average.

\subsection{Single ion and proton bulk hydration Gibbs free energy}

In Table 3 we summarize and compare to data available in literature the absolute single ion hydration Gibbs free energies $\tilde{\mu}_{\text {hyd }}^{\infty}$ computed from the droplet data of Table 1. Our anion $\tilde{\mu}_{\text {hyd }}^{\infty}$ values agree reasonably well with the data of Tissandier et $a l^{7}$ and differ slightly more from the data of Kelly et al. ${ }^{8}$ Regarding cations our $\tilde{\mu}_{\text {hyd }}^{\infty}$ values are clearly underestimated by about $10 \mathrm{kcal} \mathrm{mol}^{-1}$ compared to both the latter sets of data. That difference in the $\tilde{\mu}_{\text {hyd }}^{\infty}$ result quality between anions and cations yields also a clear difference in the absolute proton hydration Gibbs free energy $\mu_{\text {hyd }}^{\infty}\left(\mathrm{H}^{+}\right)$when estimated for both kind of ions. If we consider the Tissandier et $a l^{7}$ set of conventional ion free energies $\mu_{\text {hyd }}^{\infty, c o n}$ that differ at most by $0.1 \mathrm{kcal} \mathrm{mol}^{-1}$ from the set of Fawcett et $a l^{40}$ and that are tied to the ion and proton $\mu_{\text {hyd }}^{\infty}$ values according to

$$
\mu_{\text {hyd }}^{\infty, c o n}\left(\mathbf{X}^{z e}\right)=\tilde{\mu}_{\text {hyd }}^{\infty}\left(\mathbf{X}^{z e}\right)-z \mu_{\text {hyd }}^{\infty}\left(\mathrm{H}^{+}\right) .
$$

we get an anion-based estimate $\mu_{\text {hyd }}^{\infty}\left(\mathrm{H}^{+}\right)$of $269.2 \pm 0.1 \mathrm{kcal} \mathrm{mol}^{-1}$ that is overestimated by a few kcal mol ${ }^{-1}$ compared to the Tissandier's $^{7}$ and Kelly's ${ }^{8}$ values, 263.9 and 265.9 kcal $\mathrm{mol}^{-1}$, respectively, whereas our cation-based estimate is $255.3 \pm 0.1 \mathrm{kcal} \mathrm{mol}^{-1}$, a value that deviates more largely from the Tissandier's and Kelly's estimates, also by about $10 \mathrm{kcal}$ $\mathrm{mol}^{-1}$.

We may note first that both our cation and anion-based proton values overall agree with the results reported by earlier authors that range from 249.5 to $264 \mathrm{kcal} \mathrm{mol}^{-1} \cdot{ }^{11,15,41-43} \mathrm{We}$ may also note that the Tissandier's and Kelly's $\mu_{\text {hyd }}^{\infty}\left(\mathrm{H}^{+}\right)$values are derived by averaging data corresponding to hundreds of cation/anion pairs and that their $\mu_{\text {hyd }}^{\infty}\left(\mathrm{H}^{+}\right)$uncertainty is no less than $2 \mathrm{kcal} \mathrm{mol}^{-1} \cdot{ }^{7,8}$ It is thus not obvious to draw definitive conclusions from the data of our small ion set to assess the reliability of our computational approach. However, 
we identified a cation/water force field artifact arising from an inaccurate description of the water pair interactions among molecules belonging to the cation first and second hydration shells : the interaction energy of such water pairs is underestimated by about $1 \mathrm{kcal} \mathrm{mol}^{-1}$ by our force-field (see Supplementary Material, Section 2). As there are about 10 first/second hydration shell water pairs in bulk phase at the vicinity of both $\mathrm{Li}^{+}$and $\mathrm{NH}_{4}^{+}$, improving the description of these kind of water pair interactions should yield a priori a better agreement in the proton hydration Gibbs free energies computed from our cation and anion simulated data. Note the force fields we use for cations are built to reproduce precisely the interaction energies of cations interacting only with water molecules in their first hydration sphere and no larger cluster.

We made an attempt to remediate that cation/water force field artifact using a correction scheme similar to the one we recently proposed for anions as using our polarizable force fields. Here we reinforce the water/water interactions for molecules belonging respectively to the first and the second hydration sphere of $\mathrm{Li}^{+}$using a three body energy function whose analytical form is close to the hydrogen bonded energy term $U^{\text {lh }}$ of the water model TCPE/2013 (see Supplementary Material, Section 3). Using such a function we computed again the absolute single ion hydration Gibbs free energy $\tilde{\mu}_{\text {hyd }}^{\infty}\left(\mathbf{L i}^{+}\right)$from bulk simulations. It is then shifted to $123.4 \mathrm{kcal} \mathrm{mol}^{-1}$, in better agreement with the Tissandier's and Kelly's data. Even if the validity of such a correction scheme has to be assessed on a wider set of monovalent cations, the latter result supports the above assumption regarding the origin of the above disagreement between our computed and well accepted estimates regarding cation $\tilde{\mu}_{\text {hyd }}^{\infty}(\mathbf{X})$ data. We may thus consider that the cation force field artifact has no incidence on cation/water long range interactions and thus on the conclusions drawn above regarding the quantities $\delta \tilde{\mu}_{m \rightarrow n}$ for instance.

A priori the above cation force field artifact is not tied to the inability of our force fields to accurately model cation/water interactions but it arises mainly from a water model TCPE/2013 artifact that has no effect on modeling the properties of pure water systems. 
Hence the accurate modeling of ion hydration thermodynamic properties doesn't depend only on the quality of the description of ion/water short and medium range interactions ${ }^{44}$ but also on the quality of the description of the water/water interactions at the vicinity of ionic centers.

\subsection{The net water interface potential}

A series of of single ion hydration Gibbs free energy values have been reported ${ }^{42,45-47}$ that measure the ion bulk hydration Gibbs free energy contribution disentangled from surface effects at both the aqueous-gaz phase interface and at the boundary of the local cavity hosting the ion within water. These no surface contributions $\mu_{\text {hyd }}^{\infty, n s}$ are tied to absolute ion hydration Gibbs free energies according to

$$
\tilde{\mu}_{\text {hyd }}^{\infty}(\mathbf{X})=\mu_{\text {hyd }}^{\infty, n s}(\mathbf{X})+q_{\mathbf{X}} \Phi_{n p}
$$

here $q_{\mathbf{X}}$ is the charge of the ion $\mathbf{X} . \Phi_{n p}$ is the net interface potential, the sum of a local potential $\Phi_{l p}$ and of the water surface potential $\Delta \Phi_{b u l k}$ defined in Section 1.3. Let us consider an aqueous medium (bulk phase or a large enough finite size droplet) as a continuum medium that ignores the electrostatic charge dispersion within a water molecule. The local potential $\Phi_{l p}$ may be then interpreted as the electrostatic potential shift when crossing the boundary of the small cavity hosting an ion within the aqueous medium, not accounted for by the latter water continuum representation but arising from the water molecular charge dispersion. A priori $\Phi_{l p}$ depends on the ion nature.

As Pollard and Beck, ${ }^{16}$ we estimated a mean net potential $\tilde{\Phi}_{n p}$ from the no surface data of Marcus ${ }^{45,46}$ and from the differences in our own absolute single ion bulk hydration Gibbs free energies $\tilde{\mu}_{\text {hyd }}^{\infty}$ for cation/anion $(\mathbf{C}, \mathbf{A})$ pairs

$$
\tilde{\Phi}_{n p}=\frac{\left(\tilde{\mu}_{\text {hyd }}^{\infty}(\mathbf{C})-\tilde{\mu}_{\text {hyd }}^{\infty}(\mathbf{A})\right)-\left(\mu_{\text {hyd }}^{\infty, n s}(\mathbf{C})-\mu_{\text {hyd }}^{\infty, n s}(\mathbf{A})\right)}{2 e} .
$$


We get $\tilde{\Phi}_{n p}$ values included within $-0.30\left(\mathrm{Li}^{+} / \mathrm{Cl}^{-}\right)$and $-0.41\left(\mathrm{NH}_{4}^{+}, \mathrm{F}^{-}\right) \mathrm{V}$. If we consider the corrected value $\tilde{\mu}_{\text {hyd }}^{\infty}$ for $\mathrm{Li}^{+}, 123.4 \mathrm{kcal} \mathrm{mol}{ }^{-1}$, from our attempt to remediate the cation/water force field artifact, we get then $\tilde{\Phi}_{n p}=-0.43\left(\mathrm{Li}^{+} / \mathrm{Cl}^{-}\right)$and $-0.50\left(\mathrm{Li}^{+}, \mathrm{F}^{-}\right)$ $\mathrm{V}$. All these values agree with earlier estimates ranging from -0.40 to $-0.50 \mathrm{~V}$ (see discussions and the references cited in ${ }^{16,37}$ ) but clearly differs from the recent quantum estimates predicting a positive value for water net potential, ranging from 0.05 to $0.25 \mathrm{~V} .^{35,36}$

\subsection{The Cluster Pair Approximation for large ion/water droplets and the proton bulk hydration Gibbs free energy}

The Cluster Pair Approximation, CPA, is the assumption according to which adding a new water molecule to a large enough ion/water cluster leads to a change in the ion hydration Gibbs free energy that does not depend on the ion nature. Let us consider again an ion immersed in an aqueous medium large enough so that it can be modeled as a continuum medium. The Gibbs free energy increment to add new water molecules to that system is proportional to the square of the ion charge $q$ in line with CPA. That suggests the difference $\Delta_{\mathrm{AC}} \mu_{\text {hyd }}^{n}$ in the hydration Gibbs free energies between a cation and an anion to be constant as soon as these ions are solvated in large enough water droplets.

However modeling water as a continuum medium implicitly neglects the energy cost for a microscopic charge to cross the water surface and the cavity boundary in which the ion is hosted and arising from non zero potentials $\Delta \Phi_{b u l k}$ and $\Phi_{l p}$. Considering no surface hydration Gibbs free energies as defined in the above section and the latter two potentials, the difference in the bulk hydration Gibbs free energy between a monovalent anion $\mathbf{A}$ and cation $\mathbf{C}$ is

$$
\Delta_{\mathrm{AC}} \mu_{\mathrm{hyd}}^{\infty}=\Delta_{\mathrm{AC}} \mu_{\mathrm{hyd}}^{\infty, n s}-e\left(\Phi_{l p}^{\infty}(\mathbf{A})-\Phi_{l p}^{\infty}(\mathbf{C})\right)-2 e \cdot \Delta \Phi_{b u l k}
$$

here $\Phi_{l p}^{\infty}(\mathbf{X})$ is the local potential shift from the bulk water core to the center of the cavity 
hosting ion $\mathbf{X}$. In the droplet case that relation can be rewritten as

$$
\Delta_{\mathrm{AC}} \mu_{\text {hyd }}^{n}=\Delta_{\mathrm{AC}} \mu_{\text {hyd }}^{n, n s}-e\left(\Phi_{l p}^{n}(\mathbf{A})-\Phi_{l p}^{n}(\mathbf{C})\right)-2 e \cdot \Delta \Phi_{d}^{n},
$$

here $\Phi_{l p}^{n}(\mathbf{X})$ in the local potential for droplets. For large enough ion/water droplets, the CPA assumption yields $\Delta_{\mathrm{AC}} \mu_{\text {hyd }}^{\infty, n s}=\Delta_{\mathrm{AC}} \mu_{\text {hyd }}^{n, n s}$ and combining the above two relations yields

$$
\Delta_{\mathrm{AC}} \mu_{\text {hyd }}^{\infty}=\Delta_{\mathrm{AC}} \mu_{\text {hyd }}^{n}+e \sum_{\mathbf{X}=\mathbf{A}, \mathbf{C}} z_{\mathbf{X}}\left(\Phi_{l p}^{\infty}(\mathbf{X})-\Phi_{l p}^{n}(\mathbf{X})\right)-2 e\left(\Delta \Phi_{b u l k}-\Delta \Phi_{d}^{n}\right)
$$

Here the charge of the monovalent ion $\mathbf{X}$ is $q=z_{\mathbf{X}} e$. That relation may be considered as a generalization of the formula to compute the CPA-based estimate of the water surface potential proposed by Vlcek and co-authors. ${ }^{15}$ For large enough droplets, we expect $\Phi_{l p}^{\infty}(\mathbf{X}) \approx$ $\Phi_{l p}^{n}(\mathbf{X})$ regardless of ion $\mathbf{X}$ yielding the fundamental relation

$$
\Delta_{\mathrm{AC}} \mu_{\mathrm{hyd}}^{\infty}=\Delta_{\mathrm{AC}} \mu_{\text {hyd }}^{n}-2 e\left(\Delta \Phi_{\text {bulk }}-\Delta \Phi_{d}^{n}\right) .
$$

From the plots of the electrostatic potentials at the vicinity of our virtual atoms $\mathbf{V}_{\mathbf{a}}$ and $\mathbf{V}_{\mathbf{c}}$ in droplets (see Supplementary Material, Section 9 and Figures 8 and 9), relation (29) may be considered as exact as soon as $n=400$. Moreover according to Equation (18) the water model TCPE/2013 yields

$$
2 e\left(\Delta \Phi_{b u l k}-\Delta \Phi_{d}^{n}\right)=\frac{2 e \phi}{n^{1 / 3}}
$$

with $2 e \phi=15.3 \mathrm{kcal} \mathrm{mol}^{-1}$, a large value suggesting the difference $\left|\Delta_{\mathrm{AC}} \mu_{\text {hyd }}^{\infty}-\Delta_{\mathrm{AC}} \mu_{\text {hyd }}^{n}\right|$ to be smaller than $0.1 \mathrm{kcal} \mathrm{mol}^{-1}$ no sooner than $n=3.710^{6}$. That corresponds to a droplet whose radius is $\approx 300 \AA$. This is also supported by the data reported by Kastenholtz and Hünenberger by means of the point charge water model SPC. ${ }^{14}$ The latter authors showed a quasi linear $n^{-1 / 3}$ behavior for the droplet surface potential corresponding to a $2 e \phi$ value of about $20 \mathrm{kcal} \mathrm{mol}^{-1}$. We may also quote Vlcek and co-authors ${ }^{15}$ who showed monovalent ion hydration enthalpies to decrease slowly with the droplet size and to be roughly proportional 
to $n^{-1 / 3}$.

To check the validity of Equation (29) we may first consider the parameters $\tilde{\gamma}$ allowing to estimate the absolute ion hydration Gibbs free energies in $n>400$ droplets according to

$$
\mu_{\text {hyd }}^{\infty}(\mathbf{X})=\mu_{\text {hyd }}^{n}(\mathbf{X})-\frac{\tilde{\gamma} \mathbf{X}}{n^{1 / 3}}
$$

From the values summarized in Table 2 we note the anion parameters $\tilde{\gamma}$ to be larger than the cation ones from 7 to $10 \mathrm{kcal} \mathrm{mol}^{-1}$. However the uncertainty affecting these parameters is large, about $\pm 2.9 \mathrm{kcal} \mathrm{mol}^{-1}$ (see Supplementary Material, Section 8). The mean difference in anion/cation $\tilde{\gamma}$ values is $8.4 \pm 5.8 \mathrm{kcal} \mathrm{mol}^{-1}$, a value a priori in line with our $2 e \phi$ estimate, $15.3 \mathrm{kcal} \mathrm{mol}^{-1}$, within the error bar. Moreover we also plot in Figure 5 the quantity $\Delta \Delta_{\mathrm{AC}}^{n, \infty}=\Delta_{\mathrm{AC}} \mu_{\text {hyd }}^{\infty}-\Delta_{\mathrm{AC}} \mu_{\text {hyd }}^{n}$ for all the anion/cation pairs that may be drawn from our ion set and by considering the $4^{\text {th }}$ order power law relation (21). All the quantities $\Delta \Delta_{\mathrm{AC}}^{n, \infty}$ are decreasing functions of $n^{-1 / 3}$ and they are upper bounded by $2 e \phi \cdot n^{-1 / 3}$. That also supports the fundamental relation (30).

We plot in Figure 6 our $\Delta_{\mathrm{AC}} \mu_{\text {hyd }}^{n}$ values for droplets whose size $n$ ranges from 50 to 1000 together with those corresponding to small ion/water clusters $(n \leq 6)$ considered by Tissandier's and co-authors and Kelly's and co-authors in their original papers ${ }^{7,8}$ for all the pairs that may be built from our ions. For large droplets, we also reported on that Figure $\Delta_{\mathrm{AC}} \mu_{\text {hyd }}^{n}$ values for $\mathrm{Li}^{+}$pairs shifted by $\delta G_{\mathrm{C}}=5.8 \mathrm{kcal} \mathrm{mol}^{-1}$, the correction value arising from our attempt to remediate the cation force field artifact discussed in Section 2.4. The plots clearly show the quantity $\Delta_{\mathrm{AC}} \mu_{\text {hyd }}^{n}$ to obey two different regimes for all pairs : that quantity increases in absolute value up to $n \approx 15$ and then it linearly decreases from $n \approx 40$ to $+\infty$. That linear behavior is very close for all the ion pairs. Lastly a transition region exists for $15 \leq n \leq 40$, where the quantity $\Delta_{\mathrm{AC}} \mu_{\text {hyd }}^{n}$ should present a maximum at about $n=25$ for all our ion pairs.

The proton absolute hydration Gibbs free energy $\mu_{\text {hyd }}^{\infty}\left(\mathrm{H}^{+}\right)$can be computed from the 
differences in both anion/cation absolute $\Delta_{\mathrm{AC}} \mu_{\mathrm{hyd}}^{\infty}$ and conventional $\Delta_{\mathrm{AC}} \mu_{\mathrm{hyd}}^{\text {con, } \infty}$ hydration Gibbs free energies according to

$$
\mu_{\text {hyd }}^{\infty}\left(\mathrm{H}^{+}\right)=\frac{1}{2}\left(\Delta_{\mathrm{AC}} \mu_{\text {hyd }}^{\text {con }, \infty}-\Delta_{\mathrm{AC}} \mu_{\text {hyd }}^{\infty}\right) .
$$

According to relations (29) and (30) that may be rewritten for large enough droplets as

$$
\mu_{\text {hyd }}^{\infty}\left(\mathrm{H}^{+}\right)=\frac{1}{2}\left(\Delta_{\mathrm{AC}} \mu_{\text {hyd }}^{\text {con } \infty}-\Delta_{\mathrm{AC}} \mu_{\text {hyd }}^{n}\right)+\frac{e \phi}{n^{1 / 3}} .
$$

Both Tissandier and co-authors and Kelly and co-authors estimated the proton hydration Gibbs free energy from small ion/water clusters data assuming

$$
\mu_{\text {hyd }}^{\infty}\left(\mathrm{H}^{+}\right) \approx \frac{1}{2}\left(\Delta_{\mathrm{AC}} \mu_{\text {hyd }}^{\text {con }, \infty}-\frac{1}{c_{n}}\left(\mu_{\text {hyd }}^{n}(\mathbf{A})+\mu_{\text {hyd }}^{n}(\mathbf{C})\right)\right)
$$

here $c_{n}$ is a constant for ion/water clusters of a given size $n$. As both the water models TCPE/2013 and SPC/E predict the term $e \phi / n^{1 / 3}$ to be far from negligible up to very large droplets, it is clearly questionable to consider the latter relation as an accurate enough approximation of relation (33). In line with the earlier conclusion of Vlcek and co-authors, ${ }^{15}$ this means that the proton bulk hydration Gibbs free energy estimated from large clusters, in particular whose size $n \geq 400$, can differ significantly from well accepted estimates computed from small cluster data.

However we may note that our ion/water polarizable force fields reproduce accurately high level $a b$ initio quantum data regarding the binding energies of small ion/water clusters whose size is $n \leq 4$. As ab initio quantum approaches have been shown to be able to reproduce accurately experimental results regarding the water stepwise binding energies in small ion clusters (see among other Ref., ${ }^{31}$ for instance), we may thus reasonably consider that our polarizable force fields will provide a proton hydration Gibbs free energy value based on small cluster data close to the Tissandier's and Kelly's estimates, ${ }^{7,8}$ i.e. ranging 
from 264 to $266 \mathrm{kcal} \mathrm{mol}^{-1}$. On the other hand the proton bulk hydration Gibbs free energy estimated from our $n \geq 400$ droplet data for the pairs $\mathrm{Li}^{+} / \mathrm{F}^{-}$and $\mathrm{Li}^{+} / \mathrm{Cl}^{-}$(and accounting for the cation correction $\delta G_{\mathbf{C}}$ ) is about $265 \pm 2 \mathrm{kcal} \mathrm{mol}^{-1}$ on average. Even if more efforts are needed to understand how the latter estimate is affected by possible force field artifacts, that estimate suggests the order of magnitude of a possible difference in proton hydration Gibbs free energies as estimated from small clusters and large droplets data to be no larger than $2-3 \mathrm{kcal} \mathrm{mol}^{-1}$, i.e. a few $\mathrm{k}_{\mathrm{B}} \mathrm{T}$ unit.

\section{Conclusion}

We computed the absolute single ion bulk hydration Gibbs free energies regarding $\mathrm{Li}^{+}$, $\mathrm{NH}_{4}^{+}, \mathrm{F}^{-}$and $\mathrm{Cl}^{-}$from bulk simulations of an infinitely replicated water box in which ions are embedded and by extrapolating to their bulk limit data corresponding to finite size ion/water droplets whose water molecular size vary from $n=50$ to 1000 . We also computed the surface potential in pure water droplets whose size ranges from $n=200$ to 10000 . All our simulations were performed using our own polarizable force fields, in particular for water we consider the water model TCPE/2013. ${ }^{17}$ Our main results are

1. the TCPE/2013 surface potentials of pure water droplets obey a linear function of the droplet "radius" $R_{d}=n^{1 / 3}$ as soon as $n \geq 200$;

2. the ion hydration Gibbs free energy increment arising from additional water molecules added to a given ion/water droplet does not depend anymore from the ion nature no sooner than $n=400$ droplets;

3. for ion/water droplets corresponding to $n \geq 400$ and in line with the Born equation, the absolute ion hydration Gibbs free energies $\mu_{\text {hyd }}^{\infty}$ and the differences in anion/cation hydration Gibbs free energies $\Delta_{\mathrm{AC}} \mu_{\text {hyd }}^{n}$ are both linear functions of the inverse of the droplet radius $n^{1 / 3}$; 
4. the differences $\Delta_{\mathrm{AC}} \mu_{\text {hyd }}^{n}$ obey two different regimes in small cluster $(n \leq 15)$ and large droplet $(n \geq 40)$ domains.

Regarding absolute single ion hydration Gibbs free energies and water surface potentials in droplets, our results computed by means of point charge - point induced dipole polarizable force fields agree with earlier ones derived from standard additive point charge force fields, like those of Kastenholz and Hünenberger ${ }^{14}$ regarding water surface potentials and those of Vlcek and co-authors ${ }^{15}$ regarding ion hydration enthalpies. In particular the latter authors showed these differences to also obey two different regimes in small cluster and large droplet domains. ${ }^{15}$

We identified an artifact regarding our cation/water force fields, arising a priori from an inaccurate description of local interactions between water molecules belonging to monovalent cation first and second hydration spheres respectively, and yielding underestimated cation hydration Gibbs free energies in solution. This artifact can be remediated by adding a short range three-body energy term to improve the modeling of interactions among cation and its first/second hydration sphere water molecules. That energy term affects ion/water interactions up to a distance of about $6 \AA$ from the ionic center. Because of the size of all our droplets (for instance the radius of the $n=50$ droplet is already $>6 \AA$ ), we may thus assume the reliability of all the above conclusions.

Our results, in particular regarding the two regimes to which the differences in anion/cation hydration Gibbs free energy $\Delta_{\mathrm{AC}} \mu_{\text {hyd }}^{n}$ obey in droplets, clearly suggest that methods based on the Cluster Pair Assumption and by considering the thermodynamical properties of small ion/water clusters (whose size $n \leq 6$ ), like those proposed by Klotz, ${ }^{6}$ Tissandier's and co-authors ${ }^{7}$ and Kelly and co-authors ${ }^{8}$ to estimate the absolute proton hydration Gibbs free energy in solution $\mu_{\text {hyd }}^{\infty}\left(\mathrm{H}^{+}\right)$are questionable. Our present simulations show that taking into account larger ion/water droplets may yield a $\mu_{\text {hyd }}^{\infty}\left(\mathrm{H}^{+}\right)$value shifted by several $\mathrm{k}_{\mathrm{B}} \mathrm{T}$ units compared to $\mathrm{CPA} /$ small clusters-based estimates. 


\section{Supplementary Material}

A free trial version of our code POLARIS(MD) together with the input files allowing to perform the Gibbs free energy computations detailed in the present manuscript for the system $\mathrm{NH}_{4}^{+} / 100$ water droplet is available via a link provided as Supplementary Material. They will be soon available on the POLARIS(MD) official website (http://biodev.cea.fr/polaris/download.html). Moreover, Supplementary Material materials provide also the details of our polarizable $\mathrm{Li}^{+} /$water force field, of the water slab system used to investigate the properties of the liquid water/air interface, the formula allowing one to estimate the entropic cost arising from the perturbation of solute/solvent intramolecular vibrational properties upon ion hydration, the plots of the droplet molecular densities, the raw data and the plots corresponding to the pure water droplet surface potentials, and the surface potential of liquid water computed from our TCPE/2013 model using the "M-convention" scheme and from the pairwise water model TIP3P using the "P-convention" scheme. This material also provides files summarizing the charge and dipole quantities computed along the independent MD droplet trajectories and allowing one to compute the droplet water-vacuum surface potential.

\section{Acknowledgments}

We would like to thank Maria Reif and the reviewers for helpful discussions regarding this work. The members of the PhLAM laboratoryacknowledge support by the French government through the Program "Investissement dvenir" (LABEX CaPPA / ANR-11-LABX0005-01 and I- SITE ULNE / ANR-16-IDEX-0004 ULNE), as well as by the Ministry of Higher Education and Research, Hauts de France council and European Regional Development Fund (ERDF) through the Contrat de Projets Etat-Region (CPER CLIMIBIO). Furthermore, this work was granted access to the HPC resources of [CINES/IDRIS/TGCC] under the allocation 2016-2019 [x2016081859 and A0010801859 and A005030707] made by GENCI. 
Table 1: Single ion hydration Gibbs free energy components in kcal mol ${ }^{-1}$ of the ions $\mathrm{X}=\mathrm{Li}^{+}, \mathrm{NH}_{4}^{+}, \mathrm{F}^{-}$and $\mathrm{Cl}^{-} . \mathrm{V}$ is the virtual atom corresponding to anions or cations (see the scheme detailed in Figure 1). $\delta G_{v i b}^{g \rightarrow l}$ is a correction accounting for intramolecular vibrational frequency shifts upon hydration (see Section 1.6). $\Delta G_{\infty}$ is the droplet free energy cost $\Delta G_{n}$ defined in Figure 1 extrapolated for $n \rightarrow \infty$. The $\Delta G_{\infty}$ components $\Delta G_{\infty}^{\mathrm{sr}}$ and $\Delta G_{\infty}^{\mathrm{elec}}$ are detailed in Section 2.3. The $\mu_{\text {hyd }}^{\infty}(\mathbf{X})$ and $\mu_{\text {hyd }}^{\infty}(\mathbf{V})$ are the single ion bulk hydration Gibbs free energy for the ions and the virtual atoms (for the cation and the anion virtual atoms, the $\mu_{\text {hyd }}^{\infty}(\mathrm{V})$ values are $\mathbf{- 1 2 . 5}$ and $\mathbf{- 9 . 0} \mathrm{kcal} \mathrm{mol}^{-1}$, respectively). $q \Phi_{\text {bulk }}$ is the free energy cost for a point charge $q$ to cross the liquid water/vapor interface, i.e. the water surface potential contribution to the hydration free energy of an ion of charge $q$. The quantity $\Delta \Delta \mu$ is defined as $\Delta G_{\infty}-\left[\mu_{\text {hyd }}^{\infty}(\mathbf{V})-\mu_{\text {hyd }}^{\infty}(\mathbf{X})\right]$. Note the uncertainty affecting the quantities $\Delta G_{\infty}^{\mathrm{sr}}, \mu_{\text {hyd }}^{\infty}(\mathrm{X})$ and $\mu_{\text {hyd }}^{\infty}(\mathrm{V})$ is $0.1 \mathrm{kcal} \mathrm{mol}^{-1}$, and the uncertainties affecting the quantities $\Delta G_{\infty}^{\text {elec }}$ and $\Delta \Delta \mu$ are 0.6 and $0.8 \mathrm{kcal}$ $\mathrm{mol}^{-1}$, respectively (see text for details).

\begin{tabular}{ccccccccc}
\hline \hline Ion & $\delta G_{\text {vib }}^{g \rightarrow l}$ & $\mu_{\text {hyd }}^{\infty}(\mathbf{X})$ & $\Delta G_{\infty}^{\text {sr }}$ & $\Delta G_{\infty}^{\text {elec }}$ & $\Delta G_{\infty}$ & $\mu_{\text {hyd }}^{\infty}(\mathbf{X})-\mu_{\text {hyd }}^{\infty}(\mathbf{V})$ & $\Delta \Delta \mu$ & $q \Phi_{\text {bulk }}$ \\
\hline \hline $\mathrm{Li}^{+}$ & -0.5 & -113.1 & +9.1 & -114.6 & -105.5 & -100.6 & -4.9 & -5.3 \\
$\mathrm{NH}_{4}^{+}$ & -1.0 & -69.2 & +11.2 & -73.0 & -61.8 & -56.7 & -5.1 & -5.3 \\
$\mathrm{~F}^{-}$ & +0.4 & -103.5 & +21.8 & -110.6 & -88.8 & -103.7 & +5.9 & +5.3 \\
$\mathrm{Cl}^{-}$ & 0.0 & -77.2 & +27.0 & -90.4 & -63.2 & -68.2 & +5.0 & +5.3 \\
\hline \hline
\end{tabular}


Table 2: Parameters of the function $\mu_{\text {hyd }}^{n}(\mathbf{X})=\tilde{\mu}_{\text {hyd }}^{\infty}(\mathbf{X})+\tilde{\gamma}_{\mathbf{X}} \cdot n^{-1 / 3}$ adjusted from droplet data corresponding to $n \geq 400$, all in $\mathrm{kcal} \mathrm{mol}^{-1}$. The parameter values are provided \pm their uncertainty arising from our TI computations. Reg.: regression coefficient of the linear fitting process to adjust the parameters. The $\tilde{\mu}_{\text {hyd }}^{\infty}(\mathrm{X}) \mathrm{s}$ account for the correction term $\delta G_{v i b}^{g \rightarrow l}$, see Table 1 .

\begin{tabular}{cccc}
\hline \hline Ion & $-\tilde{\mu}_{\text {hyd }}^{\infty}$ & $\tilde{\gamma}$ & Reg. \\
\hline \hline $\mathrm{Li}^{+}$ & $118.6 \pm 0.5$ & $70.5 \pm 2.9$ & 0.999 \\
$\mathrm{NH}_{4}^{+}$ & $74.1 \pm 0.3$ & $73.1 \pm 2.9$ & 0.990 \\
$\mathrm{~F}^{-}$ & $96.4 \pm 0.3$ & $80.9 \pm 2.9$ & 0.999 \\
$\mathrm{Cl}^{-}$ & $69.8 \pm 0.3$ & $79.0 \pm 2.9$ & 0.999 \\
\hline \hline
\end{tabular}


Table 3: Absolute single ion hydration Gibbs free energies in solution and at ambient conditions, in $\mathrm{kcal} \mathrm{mol}^{-1}$. The data from our work correspond to the droplet one. $\epsilon_{\text {err }}$ : mean uncertainty affecting the free energies. $\left(^{*}\right):$ the value is a priori the same as reported by Tissandier et al. ${ }^{7}$

\begin{tabular}{|c|c|c|c|c|c|}
\hline & $\overline{\mathrm{F}^{-}}$ & $\overline{\mathrm{Cl}^{-}}$ & $\overline{\mathrm{Li}^{+}}$ & $\overline{\mathrm{NH}_{4}^{+}}$ & $\epsilon_{\text {err }}$ \\
\hline This work & $\overline{97.1}$ & 70.7 & 117.3 & 74.4 & 0.6 \\
\hline Tissandier $^{7}$ & 102.4 & 72.7 & 126.5 & 84.1 & 1.9 \\
\hline Kelly ${ }^{8}$ & 104.4 & 74.5 & 128.4 & 85.2 & $\approx 2$ \\
\hline Hünnenberger-Reif ${ }^{48}$ & 103.5 & 73.7 & 125.4 & & 1.2 \\
\hline $\mathrm{AMOEBA}^{49}$ & & 84.6 & & & 0.1 \\
\hline DFT-MD ${ }^{44}$ & 113.4 & & 119.8 & & 0.7 \\
\hline Force-Matching-MD ${ }^{50}$ & 101.4-106.2 & 68.6-71.8 & & & - \\
\hline Cluster Theory ${ }^{51}$ & 104.1 & 74.3 & 124.9 & & $(*)$ \\
\hline CC-QCT theory ${ }^{52}$ & & & 116.1 & & 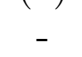 \\
\hline
\end{tabular}




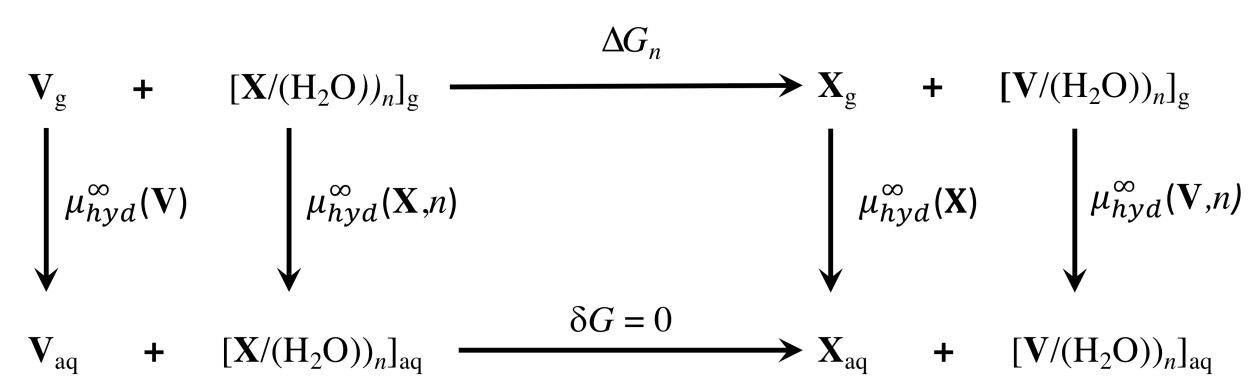

Figure 1: Thermodynamic cycle TC showing the link between bulk and finite size system single ion hydration Gibbs free energies. 

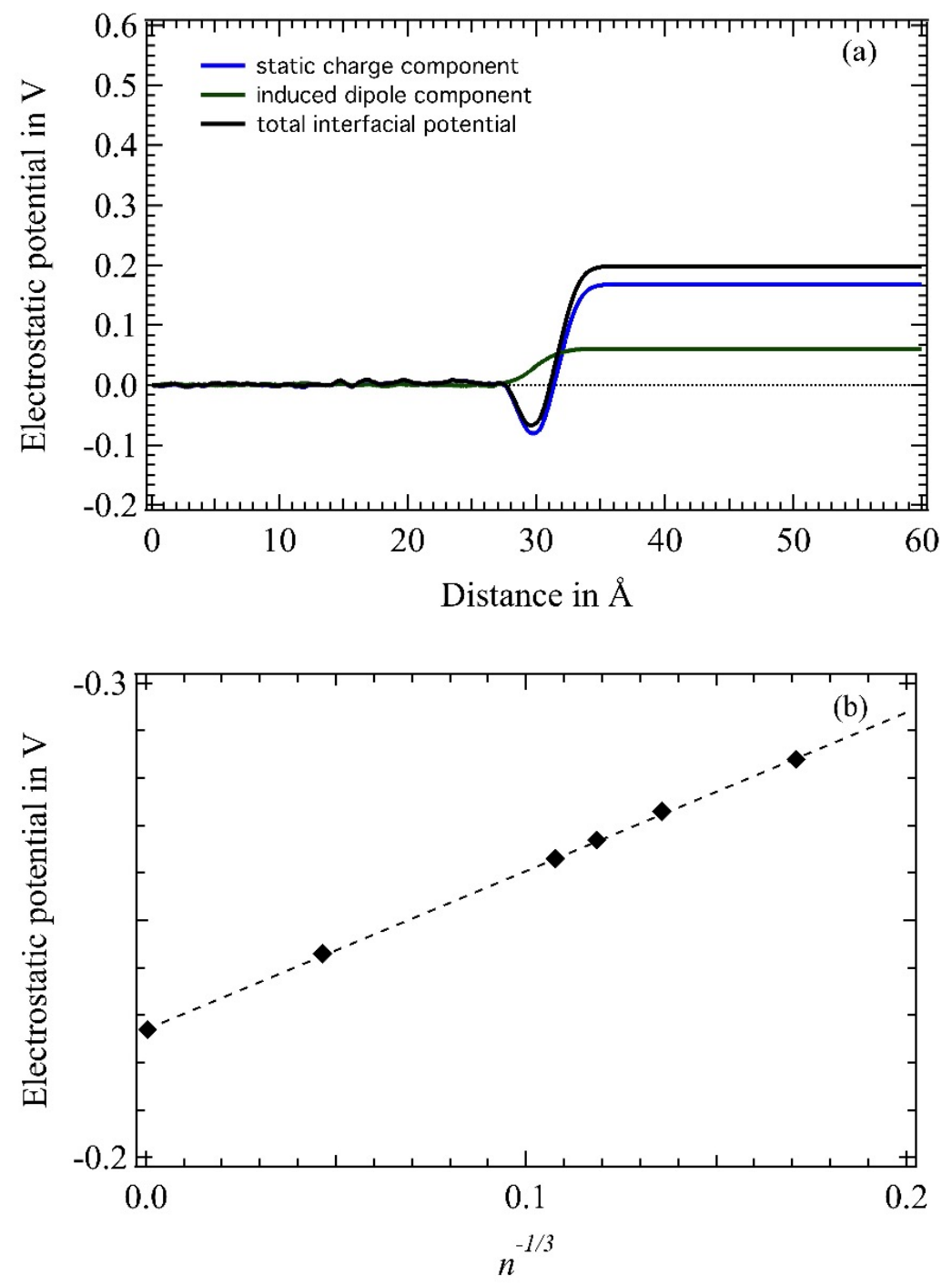

Figure 2: Water surface potential. (a): at the bulk water-vacuum interface, as a function of the distance $z$ to the interface, together with its two components computed from the atomic static charges and the induced dipole moments. (b): surface potential at water droplet surfaces wrt to $n^{-1 / 3}$ and convergence to the bulk limit (shown at $n^{-1 / 3}=0$ ). The uncertainty affecting the surface potential values is smaller than $1 \%$ of the computed values. In dashed line, the linear regression fit, the linear regression coefficient is larger than 0.999. 


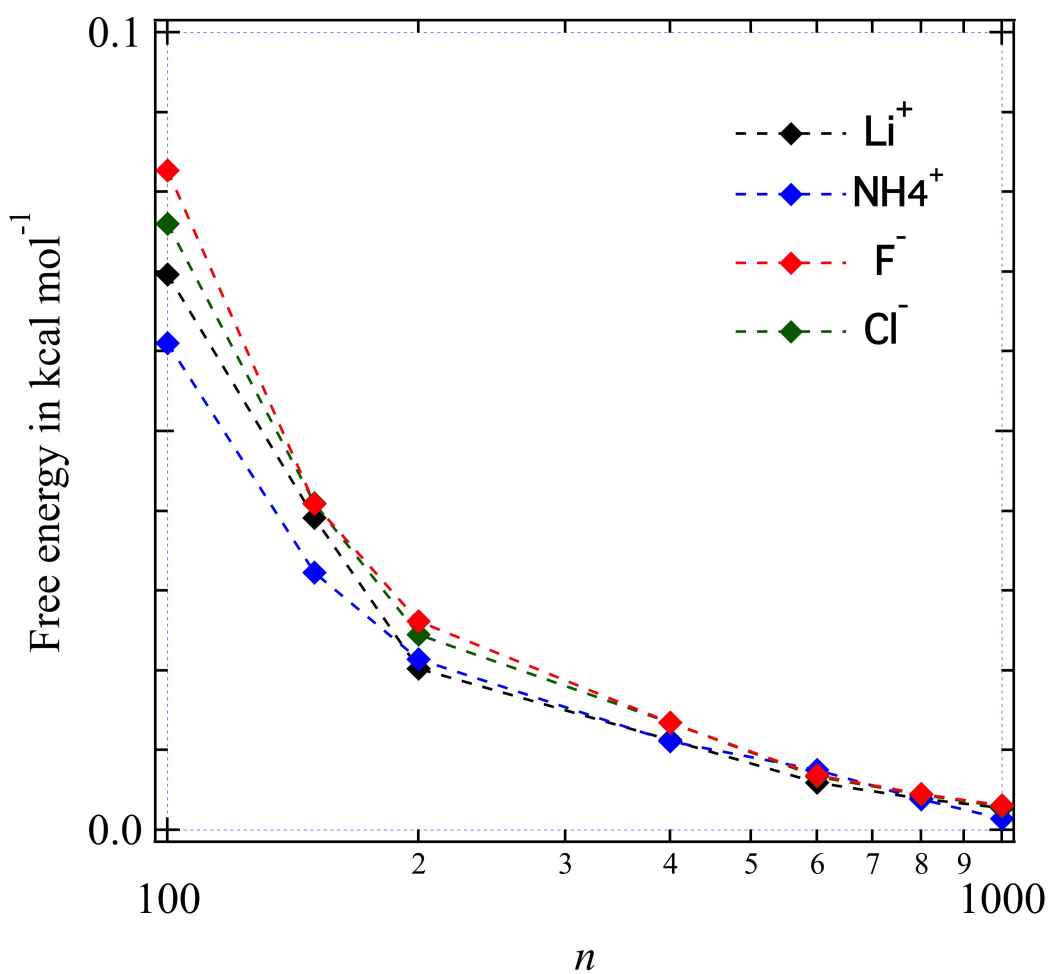

Figure 3: Evolution of the quantity $\delta \tilde{\mu}_{m \rightarrow n}$ as a function of the droplet size $n$. The uncertainty regarding the plotted values is $\leq 10^{-3} \mathrm{kcal} \mathrm{mol}^{-1}$, regardless of $n$. 


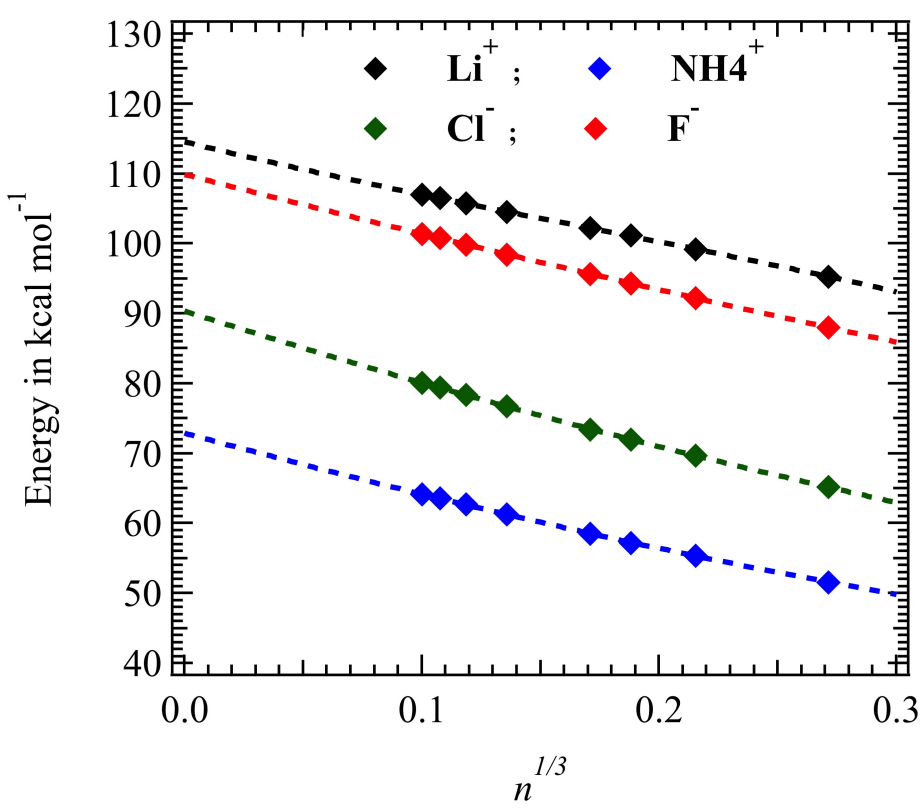

Figure 4: Droplet $\Delta G_{n}^{\text {elec }}$ values (diamonds) and the corresponding power law fitted functions (dashed lines). The $\Delta G_{n}^{\text {elec }}$ s are the electrostatic components of the free energy costs $\Delta G_{n}$ corresponding to the reaction $\mathbf{V}_{\mathrm{g}}+\left[\mathbf{X} /\left(\mathrm{H}_{2} \mathrm{O}\right)_{\mathrm{n}}\right]_{\mathrm{g}} \rightarrow \mathbf{X}_{\mathrm{g}}+\left[\mathbf{V} /\left(\mathrm{H}_{2} \mathrm{O}\right)_{\mathrm{n}}\right]_{\mathrm{g}}$, see Figure 1 and the definitions provided in Section 2.3. The uncertainty regarding the plotted values is $0.1 \mathrm{kcal}$ $\mathrm{mol}^{-1}$ (see text for details). 


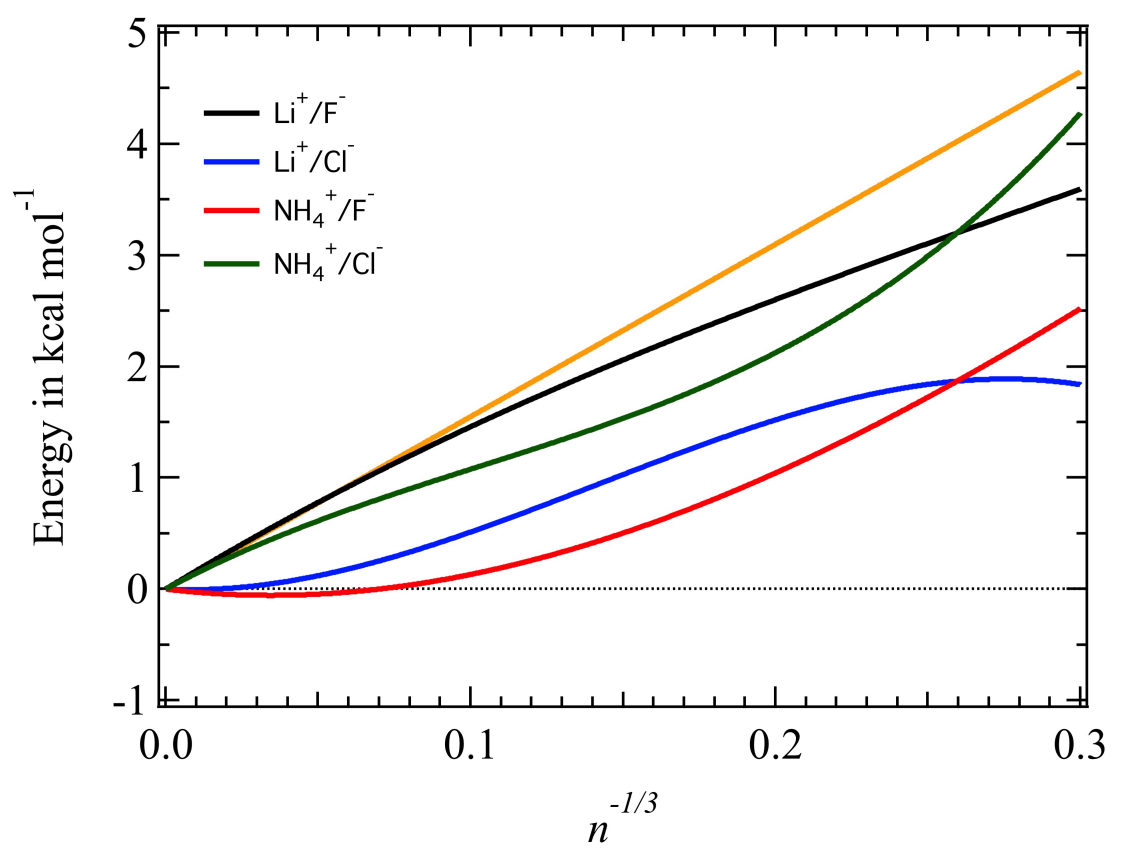

Figure 5: $\Delta \Delta_{\mathrm{AC}}^{n, \infty}$ quantities for all our anion/cation pairs as computed from $4^{\text {th }}$ order power law functions (21). In yellow, the linear function $2 e \phi \cdot n^{-1 / 3}$. 


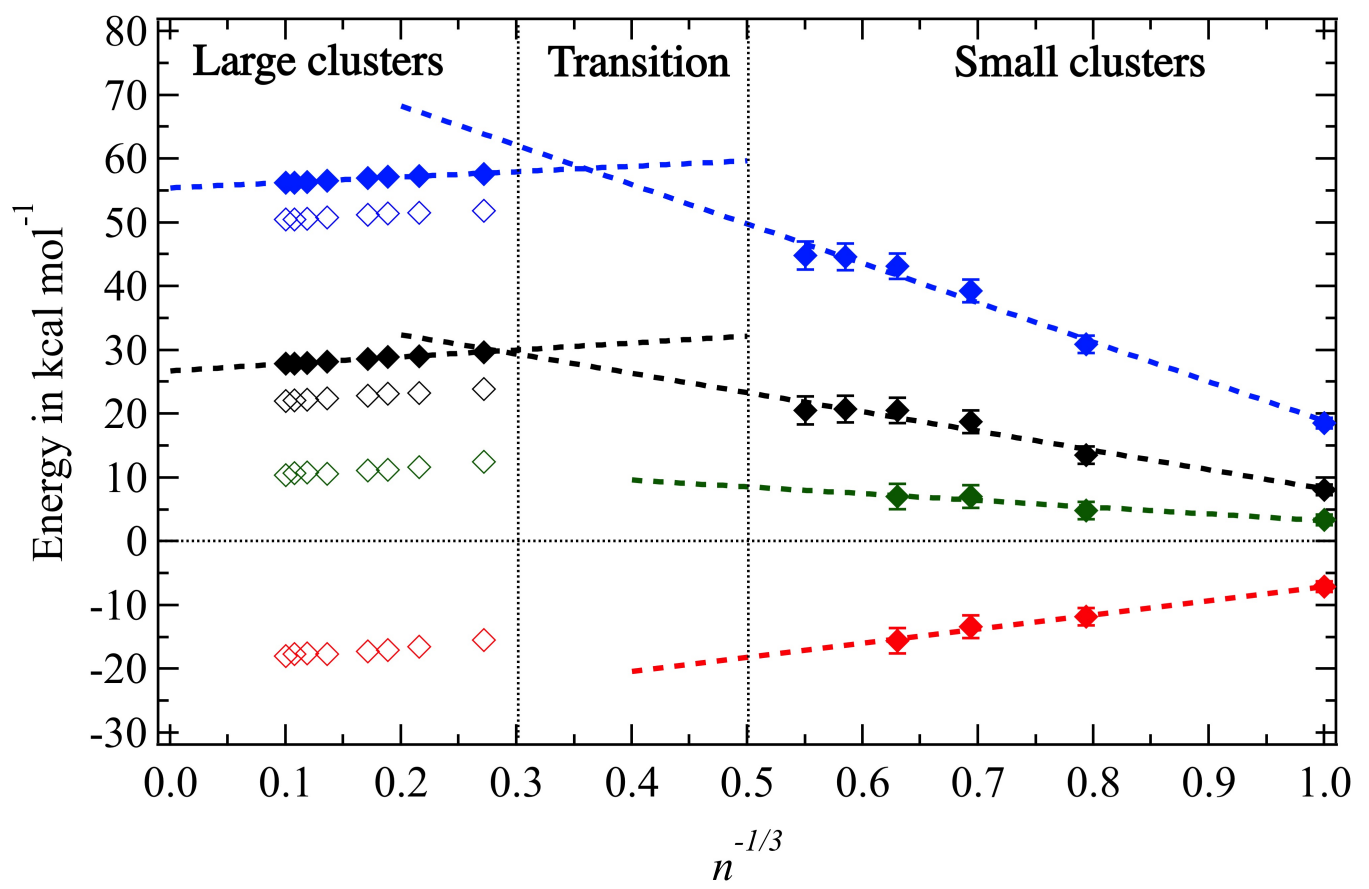

Figure 6: $\Delta_{\mathrm{AC}} \mu_{\text {hyd }}^{n}$ quantities for all our anion/cation pairs from the raw data (empty diamonds) and the corrected ones to account for the cation force field artifact (full diamonds) as computed from our ion/water droplet simulations (i.e. data corresponding to $n^{-1 / 3} \leq 0.3$, the uncertainty affecting these data is $0.1 \mathrm{kcal} \mathrm{mol}^{-1}$ ); and from the experimental data considered by Kelly and co-workers ${ }^{8}$ (for $n^{-1 / 3} \geq 0.5$ ). In dashed lines linear regression functions of the data corresponding to the small and large cluster domains. Same color labeling as in Figure 5 . 
 \\ References}

(1) Yamada, Y.; Usui, K.; Sodeyama, K.; Ko, S.; Tateyama, Y.; Yamada, A. HydrateMelt Electrolytes for High-Energy-Density Aqueous Batteries. Nature Energy 2016, 1, 16129

(2) Ariya, P. A.; Amyot, M.; Dastoor, A.; Deeds, D.; Feinberg, A.; Kos, G.; Poulain, A.; Ryjkov, A.; Semeniuk, K.; Subir, M. et al. Mercury Physicochemical and Biogeochemical Transformation in the Atmosphere and at Atmospheric Interfaces: A Review and Future Directions. Chemical Reviews 2015, 115, 3760-3802.

(3) Svensmark, H.; Enghoff, M. B.; Shaviv, N. J.; Svensmark, J. Increased Ionization Supports Growth of Aerosols into Cloud Condensation Nuclei. Nature Communications $\mathbf{2 0 1 7}, 8,2199$.

(4) Holm, R. H.; Kennepohl, P.; Solomon, E. I. Structural and Functional Aspects of Metal Sites in Biology. Chem. Rev. 1996, 96, 2239-2314.

(5) Gray, H. B. Biological Inorganic Chemistry at the Beginning of the 21st Century. Proceedings of the National Academy of Sciences of the United States of America 2003, 100, 3563-3568.

(6) Klots, C. E. Solubility of Protons in Water. The Journal of Physical Chemistry 1981, $85,3585-3588$.

(7) Tissandier, M. D.; Cowen, K. A.; Feng, W. Y.; Gundlach, E.; Cohen, M. H.; Earhart, A. D.; ; Coe, J. V.; Tuttle, T. R. T. J. The Proton's Absolute Aqueous Enthalpy and Gibbs Free Energy of Solvation from Cluster-Ion Solvation Data. The Journal of Physical Chemistry A 1998, 102, 7787-7794.

(8) Kelly, C. P.; Cramer, C. J.; Truhlar, D. G. Aqueous Solvation Free Energies of Ions and Ion: Water Clusters Based on an Accurate Value for the Absolute Aqueous Solvation 
Free Energy of the Proton. The Journal of Physical Chemistry B 2006, 110, 16066 16081.

(9) Pethica, B. A. Are Electrostatic Potentials Between Regions of Different Chemical Composition Measurable? The Gibbs-Guggenheim Principle Reconsidered, Extended and its Consequences Revisited. Phys. Chem. Chem. Phys. 2007, 9, 6253-6262.

(10) Cendagorta, J. R.; Ichiye, T. The Surface Potential of the Water-Vapor Interface from Classical Simulations. The Journal of Physical Chemistry B 2015, 119, 9114-9122.

(11) Lamoureux, G.; Roux, B. Absolute Hydration Free Energy Scale for Alkali and Halide Ions Established from Simulations with a Polarizable Force Field. The Journal of Physical Chemistry B 2006, 110, 3308-3322.

(12) Leung, K. Surface Potential at the Air-Water Interface Computed Using Density Functional Theory. The Journal of Physical Chemistry Letters 2010, 1, 496-499.

(13) Kathmann, S. M.; Kuo, I.-F. W.; Mundy, C. J.; Schenter, G. K. Understanding the Surface Potential of Water. The Journal of Physical Chemistry B 2011, 115, 43694377.

(14) Kastenholz, M. A.; Hünenberger, P. H. Computation of Methodology-Independent Ionic Solvation Free Energies from Molecular Simulations. I. The Electrostatic Potential in Molecular Liquids. The Journal of Chemical Physics 2006, 124, 124106.

(15) Vlcek, L.; Chialvo, A. A.; Simonson, J. M. Correspondence between Cluster-Ion and Bulk Solution Thermodynamic Properties: On the Validity of the Cluster-Pair-Based Approximation. The Journal of Physical Chemistry A 2013, 117, 11328-11338.

(16) Pollard, T. P.; Beck, T. L. The Thermodynamics of Proton Hydration and the Electrochemical Surface Potential of Water. The Journal of Chemical Physics 2014, 141, $18 \mathrm{C} 512$. 
(17) Réal, F.; Vallet, V.; Flament, J.-P.; Masella, M. Revisiting a Many-Body Model for Water Based on a Single Polarizable Site. From Gas Phase Clusters to Liquid and Air/Liquid Water Systems. The Journal of Chemical Physics 2013, 139, 114502.

(18) Houriez, C.; Meot-Ner (Mautner), M.; Masella, M. Simulated Solvation of Organic Ions: Protonated Methylamines in Water Nanodroplets. Convergence toward Bulk Properties and the Absolute Proton Solvation Enthalpy. The Journal of Physical Chemistry B 2014, 118, 6222-6233.

(19) Réal, F.; Severo Pereira Gomes, A.; Guerrero Martinez, Y. O.; Ayed, T.; Galland, N.; Masella, M.; Vallet, V. Structural, Dynamical, and Transport Properties of the Hydrated Halides: How Do At- and I- Bulk Properties Compare with those of the other Halides, from F- to I-. The Journal of Chemical Physics 2016, 144, 124513.

(20) Réal, F.; Trumm, M.; Schimmelpfennig, B.; Masella, M.; Vallet, V. Further Insights in the Ability of Classical Nonadditive Potentials to Model Actinide Ion-Water Interactions. Journal of Computational Chemistry 34, 707-719.

(21) Trumm, M.; Guerrero Martinez, Y. O.; Réal, F.; Schimmelpfennig, B.; Masella, M.; Vallet, V. Modeling the Hydration of Mono-Atomic Anions From the Gas Phase to the Bulk Phase: The Case of the Halide Ions $\mathrm{F}^{-}, \mathrm{Cl}^{-}$, and $\mathrm{Br}^{-}$. The Journal of Chemical Physics 2012, 136, 044509.

(22) Réal, F.; Vallet, V.; Masella, M. Improving the Description of Solvent Pairwise Interactions using Local Solute/Solvent Three-Body Functions. The Case of Halides and Carboxylates in Aqueous Environments. 2019.

(23) Houriez, C.; Meot-Ner (Mautner), M.; Masella, M. Simulated Solvation of Organic Ions II: Study of Linear Alkylated Carboxylate Ions in Water Nanodrops and in Liquid Water. Propensity for Air/Water Interface and Convergence to Bulk Solvation Properties. The Journal of Physical Chemistry B 2015, 119, 12094-12107. 
(24) Coles, J. P.; Houriez, C.; Meot-Ner (Mautner), M.; Masella, M. Extrapolating Single Organic Ion Solvation Thermochemistry from Simulated Water Nanodroplets. The Journal of Physical Chemistry B 2016, 120, 9402-9409.

(25) Houriez, C.; Meot-Ner (Mautner), M.; Masella, M. Solvation of the Guanidinium Ion in Pure Aqueous Environments: A Theoretical Study from an Ab Initio-Based Polarizable Force Field. The Journal of Physical Chemistry B 2017, 121, 11219-11228.

(26) Toukmaji, A.; Sagui, C.; Borad, J.; Darden, T. Efficient Particle-Mesh Ewald Based Approach to Fixed and Induced Dipolar Interactions. The Journal of Chemical Physics 2000, 113, 10913-10927.

(27) Feller, S. E.; Pastor, R. W.; Rojnuckarin, A.; Bogusz, S.; Brooks, B. R. Effect of Electrostatic Force Truncation on Interfacial and Transport Properties of Water. The Journal of Physical Chemistry 1996, 100, 17011-17020.

(28) Beveridge, D. L.; Schnuelle, G. W. Free Energy of a Charge Distribution in Concentric Dielectric Continua. The Journal of Physical Chemistry 1975, 79, 2562-2566.

(29) Schaaf, C.; Gekle, S. Dielectric Response of the Water Hydration Layer around Spherical Solutes. Phys. Rev. E 2015, 92, 032718.

(30) Kastenholz, M. A.; Hünenberger, P. H. Computation of Methodology-Independent Ionic Solvation Free Energies from Molecular Simulations. II. The Hydration Free Energy of the Sodium Cation. The Journal of Chemical Physics 2006, 124, 224501.

(31) Vallet, V.; Masella, M. Benchmark Binding Energies of Ammonium and AlkylAmmonium Ions Interacting with Water. Are Ammonium-Water Hydrogen Bonds Strong? Chemical Physics Letters 2015, 618, 168 - 173.

(32) Stangret, J.; Gampe, T. Ionic Hydration Behavior Derived from Infrared Spectra in HDO. The Journal of Physical Chemistry A 2002, 106, 5393-5402. 
(33) Pejov, L.; Spångberg, D.; Hermansson, K. Using MD Snapshots in ab Initio and DFT Calculations: OH Vibrations in the First Hydration Shell around Li+(aq). The Journal of Physical Chemistry A 2005, 109, 5144-5152.

(34) Śmiechowski, M. Unusual Influence of Fluorinated Anions on the Stretching Vibrations of Liquid Water. The Journal of Physical Chemistry B 2018, 122, 3141-3152.

(35) Remsing, R. C.; Baer, M. D.; Schenter, G. K.; Mundy, C. J.; Weeks, J. D. The Role of Broken Symmetry in Solvation of a Spherical Cavity in Classical and Quantum Water Models. The Journal of Physical Chemistry Letters 2014, 5, 2767-2774.

(36) Duignan, T. T.; Baer, M. D.; Schenter, G. K.; Mundy, C. J. Electrostatic solvation free energies of charged hard spheres using molecular dynamics with density functional theory interactions. The Journal of Chemical Physics 2017, 147, 161716.

(37) Pollard, T.; Beck, T. L. Quasichemical Analysis of the Cluster-Pair Approximation for the Thermodynamics of Proton Hydration. The Journal of Chemical Physics 2014, $140,224507$.

(38) O'Brien, J. T.; Williams, E. R. Effects of Ions on Hydrogen-Bonding Water Networks in Large Aqueous Nanodrops. Journal of the American Chemical Society 2012, 134, 10228-10236.

(39) Simonson, T. Accurate Calculation of the Dielectric Constant of Water from Simulations of a Microscopic Droplet in Vacuum. Chemical Physics Letters 1996, 250, $450-$ 454.

(40) Fawcett, W. R. Thermodynamic Parameters for the Solvation of Monatomic Ions in Water. The Journal of Physical Chemistry B 1999, 103, 11181-11185.

(41) Gomer, R.; Tryson, G. An Experimental Determination of Absolute Half-Cell Emf's 
and Single Ion Free Energies of Solvation. The Journal of Chemical Physics 1977, 66, $4413-4424$.

(42) Schmid, R.; Miah, A. M.; Sapunov, V. N. A New Table of the Thermodynamic Quantities of Ionic Hydration: Values and some Applications (Enthalpytropy Compensation and Born Radii). Phys. Chem. Chem. Phys. 2000, 2, 97-102.

(43) Yu, H.; Whitfield, T. W.; Harder, E.; Lamoureux, G.; Vorobyov, I.; Anisimov, V. M.; MacKerell, A. D.; Roux, B. Simulating Monovalent and Divalent Ions in Aqueous Solution Using a Drude Polarizable Force Field. J. Chem. Theor. Comp. 2010, 6, 774-786.

(44) Duignan, T. T.; Baer, M. D.; Schenter, G. K.; Mundy, C. J. Real Single Ion Solvation Free Energies with Quantum Mechanical Simulation. Chem. Sci. 2017, 8, 6131-6140.

(45) Marcus, Y. Ion Solvation; 1985.

(46) Marcus, Y. Thermodynamics of Solvation of Ions. J Chem Soc Faraday Trans 87, 2995.

(47) Asthagiri, D.; Pratt, L. R.; Ashbaugh, H. S. Absolute Hydration Free Energies of Ions, Ion-Water clusters, and Quasichemical Theory. The Journal of Chemical Physics 2003, 119, 2702-2708.

(48) Hünenberger, P.; Reif, M. Single-Ion Solvation: Experimental and Theoretical Approaches to Elusive Thermodynamic Quantities; Theoretical and Computational Chemistry Series; The Royal Society of Chemistry, 2011.

(49) Grossfield, A.; Ren, P.; Ponder, J. W. Ion Solvation Thermodynamics from Simulation with a Polarizable Force Field. Journal of the American Chemical Society 2003, 125, $15671-15682$.

(50) Li, J.; Wang, F. Accurate Prediction of the Hydration Free Energies of 20 Salts through Adaptive Force Matching and the Proper Comparison with Experimental References. The Journal of Physical Chemistry B 2017, 121, 6637-6645. 
(51) Zhan, C. G.; Dixon, D. A. Absolute Hydration Free Energy of the Proton from FirstPrinciples Electronic Structure Calculations. The Journal of Physical Chemistry A 2001, 105, 11534-11540.

764

(52) Carvalho, N. F.; Pliego, J. R. Cluster-Continuum Quasichemical Theory Calculation of the Lithium Ion Solvation in Water, Acetonitrile and Dimethyl Sulfoxide: an Absolute Single-Ion Solvation Free Energy Scale. Phys. Chem. Chem. Phys. 2015, 17, 26745 26755 . 